\title{
The Fundamental Theorem of Asset Pricing under Proportional Transaction Costs in Finite Discrete Time
}

\author{
Walter Schachermayer* \\ Vienna University of Technology
}

November 15, 2002

\begin{abstract}
We prove a version of the Fundamental Theorem of Asset Pricing, which applies to Kabanov's modelling of foreign exchange markets under transaction costs. The financial market is described by a $d \times d$ matrix-valued stochastic process $\left(\Pi_{t}\right)_{t=0}^{T}$ specifying the mutual bid and ask prices between $d$ assets.

We introduce the notion of "robust no arbitrage", which is a version of the no arbitrage concept, robust with respect to small changes of the bid ask spreads of $\left(\Pi_{t}\right)_{t=0}^{T}$.

The main theorem states that the bid-ask process $\left(\Pi_{t}\right)_{t=0}^{T}$ satisfies the robust no arbitrage condition iff it admits a strictly consistent pricing system. This result extends the theorems of Harrison-Pliska and Kabanov-Stricker pertaining to the case of finite $\Omega$, as well as the theorem of Dalang-Morton-Willinger and KabanovRásonyi-Stricker, pertaining to the case of general $\Omega$.

An example of a $5 \times 5$-dimensional process $\left(\Pi_{t}\right)_{t=0}^{2}$ shows that, in this theorem, the robust no arbitrage condition cannot be replaced by the so-called strict no arbitrage condition, thus answering negatively a question raised by Kabanov, Rásonyi and Stricker.
\end{abstract}

Key words: Arbitrage, Proportional Transaction Costs, Foreign Exchange Markets, Fundamental Theorem of Asset Pricing.

JEL classification: G10, G12, G13

AMS 2000 subject classifications: Primary 91B24; secondary 91B28, 52A20, 60H30.

*Support by the Austrian Science Foundation (FWF) under the Wittgenstein-Preis program Z36-MAT and grant SFB\#010, and by the Austrian National Bank under grant 'Jubiläumsfondprojekt Number 8699' is gratefully acknowledged. I sincerly thank U. Haböck and I. Klein for suggesting various improvements on a previous version of this paper as well as two anonymous referees for their highly informative reports. 


\section{Introduction}

The Fundamental Theorem of Asset Pricing asserts - loosely speaking — that a stochastic model of a financial market is free of arbitrage, iff there is a consistent pricing system for all derivative securities. If the price processes of the assets under consideration are expressed in terms of a traded "numeraire" asset (usually interpreted as the "bond" or the "cash account"), a consistent pricing system may as well be described by taking expectations with respect to an equivalent "risk neutral" or "martingale" probability measure.

This theorem goes back to the pioneering work of Harrison, Kreps and Pliska in the late seventies ([HK 79], [HP 81], [K 81]). It was subsequently refined by several authors (see, e.g., [DH 86], [DMW 90], [St 90], [De 92], [S 92], [KK 94], [S 94], [R 94], [DS 94], [DS 98], as well as the survey paper [S 01] for an account on the literature).

In the classical setting, one models the financial market in a "frictionless" way, i.e., without considering transaction costs. This modelling assumption clearly is a strong idealisation of the real world, and therefore models with transaction costs have been increasingly studied in the literature (e.g., [DN 90], [JK 95a], [JK 95b], [CK 96], [FH 97], [JK 99]). In particular, the work of Jouini and Kallal has already been reflected by a large audience (compare, e.g., the textbook [LW 01]).

In these models, typically there is a "bond" which serves as numeraire. The usual assumption is that, at final date $T$, all the positions in the other traded assets are liquidated, i.e., converted into units of the bond. Kabanov [K 99] pointed out that this assumption is in some applications (e.g., foreign exchange markets) unrealistic, and proposed an appealing framework to model financial markets in a numeraire-free way by considering vector-valued portfolios. This approach turns out to be conceptually interesting, even in the frictionless case, as it allows for a new look on the wealth processes, arising in financial modelling, without explicitly using stochastic integration: expressing portfolios in terms of the number of physical units of the assets, as opposed to the values of the assets in terms of some numeraire, opens new perspectives. Having said that Kabanov's framework is interesting even in the frictionless case, it goes without saying that his approach is all the more relevant to model financial markets with transaction costs.

We now recall this framework of financial modelling. In the present paper we shall slightly deviating from [K 99] — persistently use "physical units": the financial market consists of $d$ traded assets, which might be thought of as (cash-accounts in) different currencies. As stochastic base we fix a filtered probability space $\left(\Omega,\left(\mathcal{F}_{t}\right)_{t=0}^{T}, \mathbf{P}\right)$, where time $t$ runs through the finite discrete set $\{0,1, \ldots, T\}$. We shall always assume w.l.g. that $\mathcal{F}_{0}$ is trivial. The terms of trade at time $t$ are modeled via an $\mathcal{F}_{t}$-measurable $d \times d$ matrix-valued map $\omega \mapsto \Pi_{t}(\omega)$, denoting the bid and ask prices for the exchange between the $d$ assets. The entry $\pi^{i j}$ of $\Pi$ denotes the number of units of asset $i$ for which an agent can buy one unit of asset $j$ at time $t$. In other words the pair $\left\{\frac{1}{\pi^{j i}}, \pi^{i j}\right\}$ denotes the bidand ask-prices of the asset $j$ in terms of the asset $i$. We shall always suppose that the matrices $\Pi$ satisfy the requirements of the subsequent definition.

Definition 1.1 A $d \times d$-matrix $\Pi$ will be called a bid-ask-matrix, if

(i) $\pi^{i j}>0$, for $1 \leq i, j \leq d$,

(ii) $\pi^{i i}=1$, for $1 \leq i \leq d$, 
(iii) $\pi^{i j} \leq \pi^{i k} \pi^{k j}$, for $1 \leq i, j, k \leq d$.

An adapted process $\left(\Pi_{t}\right)_{t=0}^{T}$ taking values in the set of bid-ask-matrices will be called a bid-ask-process.

The interpretation of these assumptions is rather obvious (compare, e.g., [BT 00] and [BKT 01]). Condition (iii) means that, by doing a chain of exchanges (e.g., changing Dollar into Yen, and subsequently Yen into Euro), one cannot do better than by exchanging directly (e.g., Dollar into Euro). One might argue that this is a restriction of generality, but this is not really the case: if we replace conditions (ii) and (iii) by the assumption that, for $n \geq 0$ and $\left(i, i_{1}, \ldots, i_{n}, i\right)$, we have $\pi^{i i_{1}} \pi^{i_{1} i_{2}} \ldots \pi^{i_{n-1} i_{n}} \pi^{i_{n} i} \geq 1$ (which has an obvious interpretation), then we may always pass to a matrix $\widetilde{\Pi}$ satisfying the conditions of Definition 1.1 by defining

$$
\tilde{\pi}^{i j}=\min \left\{\pi^{i i_{1}} \pi^{i_{1} i_{2}} \ldots \pi^{i_{n-1} i_{n}} \pi^{i_{n} j}:\left(i_{1}, \ldots, i_{n}\right), n \geq 0\right\} .
$$

In other words, by assuming that the economic agents are sufficiently smart to take advantage of a favorable chain of exchanges, there is no loss of generality in the assumptions of Definition 1.1.

In [K 99] (compare also [KS 01b] and [KRS 01]) the bid-ask processes $\left(\Pi_{t}\right)_{t=0}^{T}$ were introduced in a somewhat indirect way. These authors start with a $d$-dimensional price process $\left(S_{t}\right)_{t=0}^{T}$, which models the prices of the $d$ assets without transaction cost in terms of some numeraire (which may be a traded asset or not). One then defines a non-negative $d \times d$-matrix $\Lambda=\left(\lambda^{i j}\right)_{1 \leq i, j \leq d}$ of transaction cost coefficients, i.e., $\lambda^{i j} \geq 0$ models the proportionally factor one has to pay in transaction costs, when exchanging the $i$ 'th into the $j$ 'th asset. Again it is natural (compare, e.g., [P 01] or [BKT 01]) to impose that, for $1 \leq i, j, k \leq d$, we have

$$
\left(1+\lambda^{i j}\right) \leq\left(1+\lambda^{i k}\right)\left(1+\lambda^{k j}\right)
$$

The transaction cost coefficients $\Lambda$ may be fixed or may depend on $t$ and $\omega$ in an adapted way. In any case, letting

$$
\pi_{t}^{i j}(\omega)=\left(1+\lambda_{t}^{i j}(\omega)\right) \frac{S_{t}^{j}(\omega)}{S_{t}^{i}(\omega)}, \quad 1 \leq i, j \leq d,
$$

one clearly obtains a bid-ask process $\left(\Pi_{t}\right)_{t=0}^{T}$.

We can rewrite (3) in matrix notation as

$$
\Pi_{t}(\omega)=\operatorname{Diag}\left(S_{t}(\omega)\right)^{-1} \circ\left(\mathbf{1}+\Lambda_{t}(\omega)\right) \circ \operatorname{Diag}\left(S_{t}(\omega)\right)
$$

where 1 denotes the $d \times d$ matrix with all entries equal to one (not to be confused with the identity matrix) and $\operatorname{Diag}\left(S_{t}(\omega)\right)$ denotes the diagonal matrix with entries $\left(S_{t}^{i}(\omega)\right)_{i=1}^{d}$ on the diagonal.

In the present paper we find it more convenient to start the modelling directly with the bid-ask process $\left(\Pi_{t}\right)_{t=0}^{T}$, without first defining $\left(S_{t}\right)_{t=0}^{T}$ and $(\Lambda)_{t=0}^{T}$ (compare, e.g., [ET 00]). From an economic point of view this seems more natural, as in a market with friction an agent is certainly faced with a bid- and an ask-price. But these prices are not necessarily decomposed into a "frictionless" price and additional transaction costs. More importantly, the possibility of decomposing a bid-ask process $\left(\Pi_{t}\right)_{t=0}^{T}$ in different ways into a frictionless 
price process plus transaction costs will lead to interesting results (see Corollary 1.10 below).

We now turn to the definition of self-financing trading strategies and related objects.

Definition 1.2 For a given bid-ask matrix $\Pi=\left(\pi^{i j}\right)_{1 \leq i, j \leq d}$ we define

- the solvency cone $\widehat{K}(\Pi)$, which is the convex cone in $\mathbb{R}^{d}$ spanned by the unit vectors $e^{i}, 1 \leq i \leq n$, and the vectors $\pi^{i j} e^{i}-e^{j}, 1 \leq i, j \leq d$,

- the cone of portfolios available at price zero, $-\widehat{K}(\Pi)$, which is the convex cone spanned by the negative unit vectors $-e^{i}, 1 \leq i \leq n$, and the vectors $-\pi^{i j} e^{i}+e^{j}$, $1 \leq i, j \leq d$,

- the space of portfolios exchangeable to zero $\widehat{F}(\Pi):=\widehat{K}(\Pi) \cap-\widehat{K}(\Pi)$, which is the linear space spanned by the vectors $\pi^{i j} e^{i}-e^{j}$, where $(i, j)$ runs through all pairs $1 \leq i, j \leq d$, such that $\pi^{i j} \pi^{j i}=1$.

The interpretation of these concepts is straightforward: the portfolios of the form $\pi^{i j} e^{i}-e^{j}$, i.e., consisting of $\pi^{i j}$ long and one short position in (physical units of) the assets $i$ and $j$, are by the definition of $\Pi$ "solvent", i.e. the $\pi^{i j}$ units of asset $i$ can be exchanged to clear the short position in asset $j$. Obviously every linear combination $\sum_{i, j} a^{i j}\left(\pi^{i j} e^{i}-e^{j}\right)$ with non-negative coefficients $a^{i j}$ also is "solvent", i.e., can be liquidated into the zero portfolio $0 \in \mathbb{R}^{d}$. A moment's reflexion reveals that these linear combinations consist precisely of the portfolios which can be liquidated into the zero portfolio. If we also allow the economic agents to "throw away" non-negative quantities of the assets, i.e., if we consider vectors $\prod_{i=1}^{n} a^{i} e^{i}$ with non-negative coefficients $a^{i}$ also to be solvent, we arrive at the above definition of the solvency cone $\widehat{K}(\Pi)$.

We remark that - except for the frictionless case, when $\pi^{i j}=\frac{1}{\pi^{i j}}$, for each pair $1 \leq i, j \leq d$ - the cone generated by the vectors $\pi^{i j} e^{i}-e^{j}$ already contains all the unit vectors $e^{i}$ : indeed, as is wellknown to every traveller exchanging foreign currencies, in the case of some market friction one may "burn" any non-negative amount of assets by doing some foolish exchanges. It is an easy exercise to mathemtically verify this intuitively obvious fact.

For the cone of portfolios available at price zero, we just turn around the point of view: starting from the zero portfolio $0 \in \mathbb{R}^{d}$, what are the portfolios which can be obtained by trading according to the terms of trade modelled by $\Pi$, and - possibly - throwing away non-negative quantities of assets? A moment's reflexion reveals that this cone simply equals $-\widehat{K}(\Pi)$.

Finally, the space $\widehat{F}(\Pi)$ describes the vector space formed by those portfolios, which can be converted into the zero portfolio and vice versa.

We have used the "hat" in Definition 1.2 above to keep in line with the notation in [K 99], [KS 01b], [KRS 01] where the "hat notation" is systematically used to indicate that the terms refer to physical units of the assets (as opposed to the values expressed in terms of a numeraire, in which case the hat is dropped). We note, however, that this is only done for the sake of coherence of notation, as in the present paper we are persistently using the numeraire-free approach of counting in physical units, so that this distinction is not relevant here. 
Definition 1.3 For a given bid-ask matrix $\Pi$ we denote — similarly as in [K 99] — by $\widehat{K}^{*}(\Pi)$ the polar of the cone $-\widehat{K}(\Pi)$, i.e.,

$$
\begin{aligned}
\widehat{K}^{*}(\Pi) & =\left\{w \in \mathbb{R}^{d}:\langle v, w\rangle \leq 0, \text { for } v \in-\widehat{K}(\Pi)\right\} \\
& =\left\{w \in \mathbb{R}^{d}:\langle v, w\rangle \geq 0, \text { for } v \in \widehat{K}(\Pi)\right\} .
\end{aligned}
$$

We call the elements $w \in \widehat{K}^{*}(\Pi) \backslash\{0\}$ (resp. $w$ in the relative interior of $\widehat{K}^{*}(\Pi)$ ) price systems consistent (resp. strictly consistent) with the bid-ask matrix $\Pi$.

As the convex cone $\widehat{K}(\Pi)$ is generated by the vectors $e^{i}, 1 \leq i \leq n$ and $\pi^{i j} e^{i}-e^{j}$, $1 \leq i, j \leq d$, the polar $\widehat{K}^{*}(\Pi)$ of $-\widehat{K}(\Pi)$ is the intersection of $\mathbb{R}_{+}^{d}$ with the halfspaces $\left\{w \in \mathbb{R}^{d}:\left\langle\pi^{i j} e^{i}-e^{j}, w\right\rangle \geq 0\right\}$, which yields the following equivalent definition of $\widehat{K}^{*}(\Pi)$ :

$$
\widehat{K}^{*}(\Pi)=\left\{w \in \mathbb{R}_{+}^{d}: \pi^{i j} w^{i} \geq w^{j}, \text { for } 1 \leq i, j \leq d\right\} .
$$

The cone $\widehat{K}^{*}(\Pi)$ has an appealing economic interpretation, to which we tried to allude by the term "consistent price system". A vector $w \neq 0$ is in $\widehat{K}^{*}(\Pi)$ iff it defines a frictionless pricing system for the assets $1, \ldots, d$, which is consistent with the bid-ask-matrix $\Pi$ in the following sense: if the price of asset $i$ (denoted in terms of some numeraire) equals $w^{i}$, then the frictionless exchange rates, denoted by $\tau^{i j}$, clearly equal

$$
\tau^{i j}=\frac{w^{j}}{w^{i}}, \quad 1 \leq i, j \leq d
$$

Hence $w$ satisfies the inequalities $\frac{w_{j}}{w_{i}} \leq \pi^{i j}$ appearing in (5) iff $\tau^{i j} \leq \pi^{i j}$, for $1 \leq i, j \leq d$; equivalently this may be characterised by the existence of transaction cost coefficients $\lambda^{i j} \geq 0$, such that $\pi^{i j}=\left(1+\lambda^{i j}\right) \tau^{i j}$.

Passing to the notion of a strictly consistent price system: it is an easy and instructive exercise to verify that, with the above identifications, $w \in \widehat{K}^{*}(\Pi)$ is in the relative interior of $\widehat{K}^{*}(\Pi)$ iff, for each pair $1 \leq i, j \leq d$ with $\pi^{i j} \pi^{j i}>1$, we have $\pi^{i j}>\frac{w^{j}}{w^{i}}$ and $\pi^{j i}>\frac{w^{i}}{w^{j}}$ (compare Proposition A.5 below). Speaking more economically: a consistent price system $w=\left(w^{i}\right)_{i=1}^{d}$ is strictly consistent iff, for all $1 \leq i, j \leq d$, the exchange rate $\tau^{i j}=\frac{w^{j}}{w^{i}}$ is in the relative interior of the bid-ask spread $\left[\frac{1}{\pi^{j i}}, \pi^{i j}\right]$.

Finally, we note that the matrices $\tau=\left(\tau^{i j}\right)_{1 \leq i, j \leq d}$ obtained from $w \in \mathbb{R}_{+}^{d} \backslash\{0\}$ via (6) determine $w$, up to a multiplicative (strictly positive) factor. Economically speaking, this reflects the liberty in the choice of numeraire, in terms of which the prices $w^{1}, \ldots, w^{d}$ are quoted.

Definition 1.4 An $\mathbb{R}^{d}$-valued adapted process $\vartheta=\left(\vartheta_{t}\right)_{t=0}^{T}$ is called a self-financing portfolio process for the bid-ask process $\left(\Pi_{t}\right)_{t=0}^{T}$, if the increments

$$
\xi_{t}(\omega):=\vartheta_{t}(\omega)-\vartheta_{t-1}(\omega)
$$

take their values almost surely in the cone $-\widehat{K}\left(\Pi_{t}(\omega)\right)$ of portfolios available at price zero, for all $t=0, \ldots, T$ (we always define $\vartheta_{-1} \equiv 0$ ).

For $0 \leq t \leq T$ we denote by $\widehat{A}_{t}$ the convex cone in $L^{0}\left(\Omega, \mathcal{F}_{t}, \mathbf{P} ; \mathbb{R}^{d}\right)$ formed by the random variables $\vartheta_{t}$, where $\vartheta=\left(\vartheta_{i}\right)_{i=0}^{T}$ runs through the self-financing portfolio processes. 
$\widehat{A}_{T}$ may be interpreted as the set of random portfolios at time $T$, expressed in numbers of physical units of assets $1, \ldots, d$, which may be obtained by performing the "exchange operations" $\left(\xi_{t}\right)_{t=0}^{T}$. Therefore $\widehat{A}_{T}$ (and, similarly, $\widehat{A}_{t}$ for $\left.0 \leq t \leq T\right)$ may be interpreted as the "hedgeable claims", expressed in terms of physical units, starting from an initial endowment $0 \in \mathbb{R}^{d}$. We note that we use the term "self-financing" in a broad sense, as we also allow the agents to "throw away" non-negative quantities of the assets. Hence the present notation of self-financing strategies also allows for possible consumption.

The reader might be surprised, that the definition of self-financing processes is so simple and does not involve any stochastic integral. The reason is that the changes of (relative) prices of stocks are already absorbed into the definition of the solvency cones $\widehat{K}_{t}(\omega)$. As we mentioned above, we find this feature in the approach of Kabanov, Rásonyi and Stricker ([K 99], [KS 01b], [KRS 01]) very appealing from a conceptual point of view.

We now define the notion of consistent price processes $\left(Z_{t}\right)_{t=0}^{T}$, which is dual to the notion of self-financing portfolio processes; it plays a similar role as the notion of an equivalent martingale measure in the frictionless theory.

Definition 1.5 An adapted $\mathbb{R}_{+}^{d}$-valued process $Z=\left(Z_{t}\right)_{t=0}^{T}$ is called a consistent (resp. strictly consistent) price process for the bid-ask process $\left(\Pi_{t}\right)_{t=0}^{T}$, if $Z$ is a martingale under $\mathbf{P}$, and $Z_{t}(\omega)$ lies in $\widehat{K}_{t}^{*}(\omega) \backslash\{0\}$ (resp. in the relative interior of $\widehat{K}_{t}^{*}(\omega)$ ) a.s., for each $t=0, \ldots, T$.

The idea behind this notion is that, for each $t$ and $\omega$, the vector $Z_{t}(\omega)=$ $\left(Z_{t}^{1}(\omega), \ldots, Z_{t}^{d}(\omega)\right)$ defines prices for the assets $1, \ldots, d$, which are consistent (resp. strictly consistent) with the bid-ask matrix $\Pi_{t}(\omega)$. These prices should, in addition, be intertemporarily related, such that, for each asset $i$, its price at time $t$, denoted by $Z_{t}^{i}(\omega)$, equals the conditional expectation of its price $Z_{t+1}^{i}(\omega)$ at time $t+1$.

The above economic interpretation can also be found in [CK 96] in the one-dimensional case, and in [BT 00] in the present context (with constant transaction cost matrix $\Lambda$ ). The above notion was identified in [KS 01b] and [KRS 01] as a key concept in the theory (but without elaborating on the economic interpretation) as martingales $Z$ taking their values almost surely in $\widehat{K}_{t}(\omega)^{*} \backslash\{0\}$ (resp. in the relative interior of $\left.\widehat{K}_{t}(\omega)^{*}\right)$. We could not resist to baptize this notion as "(strictly) consistent price processes".

How is its relation to the more traditional notion of an equivalent martingale measure? Given a consistent price process $Z$, fix a traded asset as numeraire, say the first asset, and rewrite $Z$ as the prices $S$ of the other assets, expressed in terms of the numeraire, i.e.,

$$
S_{t}=\left(1, \frac{Z_{t}^{2}}{Z_{t}^{1}}, \ldots, \frac{Z_{t}^{d}}{Z_{t}^{1}}\right), \quad t=0, \ldots, T .
$$

We also define the measure $\mathbf{Q}$ on $\mathcal{F}_{T}$ by

$$
\frac{d \mathbf{Q}}{d \mathbf{P}}=\frac{Z_{T}^{1}}{Z_{0}^{1}}
$$

As $\left(Z_{t}^{1}\right)_{t=0}^{T}$ is assumed to be a strictly positive martingale, the measure $\mathbf{Q}$ is a probability measure on $\mathcal{F}_{T}$, equivalent to $\mathbf{P}$, and the Radon-Nikodym derivative of the restriction of $\mathbf{Q}$ to $\mathcal{F}_{t}$ with respect to $\mathbf{P}$ equals $\mathbf{E}\left[\frac{d \mathbf{Q}}{d \mathbf{P}} \mid \mathcal{F}_{t}\right]=\frac{Z_{t}^{1}}{Z_{0}^{1}}$. 
The process $\left(S_{t}\right)_{t=0}^{T}$ is a Q-martingale as, for $j=1, \ldots d$,

$$
\begin{aligned}
\mathbf{E}_{\mathbf{Q}}\left[S_{t}^{j}-S_{t-1}^{j} \mid \mathcal{F}_{t-1}\right] & =\mathbf{E}_{\mathbf{Q}}\left[\left(\frac{Z_{t}^{j}}{Z_{t}^{1}}-\frac{Z_{t-1}^{j}}{Z_{t-1}^{1}}\right) \mid \mathcal{F}_{t-1}\right] \\
& =\mathbf{E}_{\mathbf{P}}\left[\left(\frac{Z_{t}^{j}}{Z_{t}^{1}}-\frac{Z_{t-1}^{j}}{Z_{t-1}^{1}}\right) \frac{Z_{t}^{1}}{Z_{t-1}^{1}} \mid \mathcal{F}_{t-1}\right] \\
& =\frac{1}{Z_{t-1}^{1}} \mathbf{E}_{\mathbf{P}}\left[Z_{t}^{j} \mid \mathcal{F}_{t-1}\right]-\frac{Z_{t-1}^{j}}{Z_{t-1}^{1}} \mathbf{E}_{\mathbf{P}}\left[\frac{Z_{t}^{1}}{Z_{t-1}^{1}} \mid \mathcal{F}_{t-1}\right] \\
& =\frac{Z_{t-1}^{j}}{Z_{t-1}^{1}}-\frac{Z_{t-1}^{j}}{Z_{t-1}^{1}}=0
\end{aligned}
$$

Hence (9) defines an equivalent measure, under which the stock price process $S$ is a martingale.

Of course, this correspondence also works in the other direction: start with an $\mathbb{R}_{+}^{d}$ valued price process $S$ of $d$ assets, expressed in terms of the first asset, which serves as numeraire, i.e.,

$$
S_{t}=\left(1, S_{t}^{2}, \ldots, S_{t}^{d}\right), \quad t=0, \ldots, T,
$$

and assume that $S$ is a martingale under a probability measure $\mathbf{Q}$ equivalent to $\mathbf{P}$. Define $Z_{t}^{1}$ by

$$
Z_{t}^{1}=\mathbf{E}\left[\frac{d \mathbf{Q}}{d \mathbf{P}} \mid \mathcal{F}_{t}\right]
$$

and

$$
Z_{t}^{j}=S_{t}^{j} Z_{t}^{1}, \quad \text { for } j=2, \ldots, n .
$$

Then $Z=\left(Z^{1}, \ldots, Z^{d}\right)$ is a $\mathbf{P}$-martingale and takes its values in $\widehat{K}_{t}^{*}(\omega)$, where $\widehat{K}_{t}(\omega)=$ $\widehat{K}\left(\Pi_{t}(\omega)\right)$ and $\Pi_{t}(\omega)$ is induced by the (frictionless) bid-ask matrix $\pi_{t}^{i j}(\omega)=\frac{S_{t}^{j}(\omega)}{S_{t}^{i}(\omega)}$. As $\Pi_{t}(\omega)$ is frictionless, the cone $\widehat{K}^{*}\left(\Pi_{t}(\omega)\right)$ is one-dimensional, so that $Z$ is automatically strictly consistent.

The attentive reader has noticed that this relation between $S$ and $Z$ is one-to-one, up to a multiplicative factor for $Z$. We also remark that the choice of the first asset as numeraire is not canonical at all (this is precisely the motivation behind the present numeraire-free modelling) and can be replaced by choosing any other strictly positive traded asset.

We now recall the definitions of the notion of "no arbitrage" as given in [KS 01b] and [KRS 01].

Definition 1.6 (compare [KS 01b] and [KRS 01])

(i) The bid-ask process $\left(\Pi_{t}\right)_{t=0}^{T}$ satisfies no arbitrage property $(N A)$ if

$$
\widehat{A}_{T} \cap L^{0}\left(\Omega, \mathcal{F}_{T}, \mathbf{P} ; \mathbb{R}_{+}^{d}\right)=\{0\}
$$

(ii) The bid-ask process $\left(\Pi_{t}\right)_{t=0}^{T}$ satisfies the strict no arbitrage property $\left(N A^{s}\right)$ if, for every $t=0, \ldots, T$,

$$
\widehat{A}_{t} \cap L^{0}\left(\Omega, \mathcal{F}_{t}, \mathbf{P} ; \widehat{K}_{t}\right) \subseteq L^{0}\left(\Omega, \mathcal{F}_{t}, \mathbf{P} ; \widehat{F}_{t}\right)
$$


Equality (14) has the usual intuitive interpretation of the impossibility to "make something out of nothing": if a self-financing portfolio process $\vartheta=\left(\vartheta_{t}\right)_{t=0}^{T}$ is such that $\vartheta_{T}^{i} \geq 0$ almost surely, for each $i=1, \ldots, d$, then we necessarily have $\vartheta_{T} \equiv 0$.

This notion was defined in [KS 01b] and [KRS 01] — in a formally different but equivalent way - under the name of "weak no arbitrage condition". As this concept appears to us as the natural counterpart to the usual no arbitrage condition in the frictionless setting, and also in view of Definition 1.8 and Theorem 1.7 below, we propose to call this property simply "no arbitrage".

The interpretation of the "strict no arbitrage property" $\left(\mathrm{NA}^{\mathrm{s}}\right)$ is more technical: fix $t \in\{0, \ldots, T\}$ and consider a self-financing portfolio process $\vartheta=\left(\vartheta_{i}\right)_{i=0}^{T}$ such that $\vartheta_{t} \in$ $L^{0}\left(\Omega, \mathcal{F}_{t}, \mathbf{P} ; \widehat{K}_{t}\right)$, i.e., $\vartheta_{t}(\omega)$ lies almost surely in the solvency cone $\widehat{K}_{t}(\omega)$. This means that we could liquidate at time $t$ the position $\vartheta_{t}(\omega)$, to end up with the zero portfolio. In more mathematical terms this may be rephrased, that the process $\left(\vartheta_{0}, \ldots, \vartheta_{t-1}, 0, \ldots, 0\right)$ is self-financing. The assumption $\left(\mathrm{NA}^{\mathrm{s}}\right)$ asserts that, for each $0 \leq t \leq T$ this is only possible if $\vartheta_{t}(\omega)$ takes its values in the space $\widehat{F}_{t}(\omega)$ almost surely, i.e., the portfolio $\vartheta_{t}(\omega)$ may be converted to zero and vice versa.

We refer to [KS 01b] and [KRS 01] for more information on this concept and, in particular, for an example, showing that the validity of (15), for every $t=0, \ldots, T$, cannot be replaced by its validity for $t=T$ only.

In $[\mathrm{KS} 01 \mathrm{~b}]$ the following extension of the Harrison-Pliska Theorem to the present setting was obtained: under the assumption that $\Omega$ is finite, a bid-ask process $\Pi=\left(\Pi_{t}\right)_{t=0}^{T}$ satisfies the no arbitrage condition, iff there is a consistent price system $Z=\left(Z_{t}\right)_{t=0}^{T}$ for П. A characterisation of the strict no arbitrage condition for the case of finite $\Omega$ was also obtained. In [KRS01] it was shown that for general probability spaces $\Omega$ the strict no arbitrage condition is equivalent to the existence of a strictly consistent price system, under an additional assumption. The additional assumption is called "efficient friction" and requires that $\widehat{F}_{t}(\omega)=\{0\}$, a.s., for all $t=0, \ldots, T$. An interesting and relevant extension of this result was obtained by I. Penner [P 01].

It was asked in [KRS 01] whether the additional assumption of "efficient friction" can be dropped. We give in section 3 below an example of a bid-ask process $\Pi$, with $d=5$ and $T=2$, showing that - in general - the answer to this question is no. On the other hand, and more importantly, we shall presently define a slight strengthing of the notion $\left(\mathrm{NA}^{\mathrm{s}}\right)$, called the robust no arbitrage condition $\left(\mathrm{NA}^{\mathrm{r}}\right)$. Admitting this notion (Definition 1.9 below) for a moment, we may formulate the subsequent theorem, which is the main result of the paper.

Theorem 1.7 A bid ask process $\Pi=\left(\Pi_{t}\right)_{t=0}^{T}$ satisfies the robust no arbitrage condition $\left(N A^{r}\right)$, if and only if it admits a strictly consistent pricing process $Z=\left(Z_{t}\right)_{t=0}^{T}$.

In [KRS 02], which was written on the basis of a working version of the present paper, this theorem is also presented in the more abstract framwork described in terms of random polyhedral cones.

We still have to define the "robust no arbitrage" condition. We first need a preliminary notion.

Definition 1.8 For two $d \times d$ bid-ask matrices $\Pi$, $\widetilde{\Pi}$, we say that $\widetilde{\Pi}$ has smaller bid-ask spreads than $\Pi$ if, for all $1 \leq i, j \leq d$, the bid-ask interval $\left[\frac{1}{\tilde{\pi}^{j i}}, \tilde{\pi}^{i j}\right]$ is contained in the 
relative interior of $\left[\frac{1}{\pi^{j i}}, \pi^{i j}\right]$.

In other words, we require that

(i) for each pair $1 \leq i, j \leq d$, such that $\pi^{i j} \pi^{j i}=1$, we have $\pi^{i j}=\widetilde{\pi}^{i j}$ and $\pi^{j i}=\widetilde{\pi}^{j i}$,

(ii) for each pair $1 \leq i, j \leq d$, such that $\pi^{i j} \pi^{j i}>1$, we have $\pi^{i j}>\widetilde{\pi}^{i j}$ and $\pi^{j i}>\widetilde{\pi}^{j i}$.

We say that the bid-ask process $\left(\widetilde{\Pi}_{t}\right)_{t=0}^{T}$ has smaller bid-ask spreads than the bid-ask process $\left(\Pi_{t}\right)_{t=0}^{T}$ if, for each $t=0, \ldots, T$ and almost each $\omega \in \Omega$, the matrix $\widetilde{\Pi}_{t}(\omega)$ has smaller bid-ask spreads than the matrix $\Pi_{t}(\omega)$.

The passage from $\Pi$ to $\widetilde{\Pi}$ may be thought of as a "discount on the bid-ask spreads offered by the broker"; indeed, $\widetilde{\Pi}$ has smaller bid-ask intervals than $\Pi$ iff for each pair $i \neq j$ there ist $\varepsilon^{i j}>0$ such that $\tilde{\pi}^{i j}=\pi^{i j}-\varepsilon^{i j}\left(\pi^{i j}-\frac{1}{\pi^{j i}}\right)$. Of course, if $\pi^{i j}=\frac{1}{\pi^{j i}}$, there is no room for giving a discount - at least not in an arbitrage-free way - and we must have $\tilde{\pi}^{i j}=\pi^{i j}$. This leads to the — notationally awkward - fact, that, for a frictionless bid-ask matrix $\Pi$, the matrix $\Pi$ itself has "smaller bid-ask spreads" than $\Pi$.

Note that, if the bid-ask process $\widetilde{\Pi}$ has smaller bid-ask spreads than $\Pi$, each selffinancing process $\vartheta=\left(\vartheta_{t}\right)_{t=0}^{T}$ for $\Pi$ is, a fortiori, self-financing for $\widetilde{\Pi}$ too.

Definition 1.9 A bid-ask process $\left(\Pi_{t}\right)_{t=0}^{T}$ satisfies the robust no arbitrage condition $\left(\mathrm{NA}^{\mathrm{r}}\right)$ if there is a bid-ask process $\left(\widetilde{\Pi}_{t}\right)_{t=0}^{T}$ with smaller bid-ask spreads, such that $\left(\widetilde{\Pi}_{t}\right)_{t=0}^{T}$ satisfies the no arbitrage condition (NA).

Some comments on this notion are in order: firstly note that, in the frictionless case, i.e., if $\pi_{t}^{i j} \pi_{t}^{j i}=1$ a.s. for all $1 \leq i, j \leq d$ and $t=0, \ldots, T$, the notions of robust no arbitrage and no arbitrage are equivalent (as well as the notion of strict no arbitrage), and coincide with the classical notion of no arbitrage as defined in [HP 81] and [DMW 90].

In the case of market friction, i.e., when $\pi^{i j}>\frac{1}{\pi^{j i}}$ for some pairs $1 \leq i, j \leq d$, the idea of the "robust no arbitrage condition" $\left(\mathrm{NA}^{\mathrm{r}}\right)$ is, that there is still room for the broker to "offer some discount", without creating an arbitrage possibility. A similar notion was introduced and analyzed under the name of "epsilon no arbitrage" in [KPT 99].

Turning to the negation of the concept $\left(\mathrm{NA}^{\mathrm{r}}\right)$ : a bid-ask process $\left(\Pi_{t}\right)_{t=0}^{T}$ violates the "robust no arbitrage" condition, if, for every $\left(\widetilde{\Pi}_{t}\right)_{t=0}^{T}$ with smaller bid-ask spreads we may find an arbitrage. The interpretation of the negation of $\left(\mathrm{NA}^{\mathrm{r}}\right)$ therefore is, that, whenever the agent has the possibility to enter into a market with more competitive transaction costs, she can make an arbitrage (compare also the economic interpretation of "weak arbitrage opportunities" as given in [KRS 01] in the paragraph following the definition of "efficient friction" (EF)).

Finally, we state how Theorem 1.7 allows to decompose an arbitrage free bid-ask price process into an arbitrage-free price process plus transaction costs.

Corollary 1.10 For a bid-ask process $\Pi=\left(\Pi_{t}\right)_{t=0}^{T}$ satisfying the robust no arbitrage condition $\left(N A^{r}\right)$ there is an $\mathbb{R}_{+}^{d}$-valued price process $S=\left(S_{t}^{1}, \ldots, S_{t}^{d}\right)_{t=0}^{T}$ which is a martingale under some probability measure $\mathbf{Q}$ equivalent to $\mathbf{P}$, and an adapted process $\Lambda=\left(\Lambda_{t}\right)_{t=0}^{T}$ with values in the set of non-negative transaction costs matrices satisfying (2), such that

$$
\pi_{t}^{i j}(\omega)=\left(1+\lambda_{t}^{i j}(\omega)\right) \frac{S_{t}^{j}(\omega)}{S_{t}^{i}(\omega)}, \quad 1 \leq i, j \leq d .
$$


Proof Admitting Theorem 1.7 there is a strictly consistent price system $Z=\left(Z_{t}\right)_{t=0}^{T}$. Define $S$ and $\mathbf{Q}$ as in (8) and (9) and $\Lambda$ via (3).

As $Z_{t} \in \widehat{K}^{*}\left(\Pi_{t}\right)$ a.s., we have $Z_{t}^{j} \leq \pi_{t}^{i j} Z_{t}^{i}$, so that equation (3) gives

$$
1+\lambda_{t}^{i j}=\frac{\pi_{t}^{i j} S_{t}^{i}}{S_{t}^{j}}=\frac{\pi_{t}^{i j} Z_{t}^{i}}{Z_{t}^{j}} \geq 1
$$

so that $\Lambda$ indeed takes its values in the non-negative matrices. The verification of property (2) also is straight-forward:

$$
\begin{aligned}
\left(1+\lambda_{t}^{i k}\right)\left(1+\lambda_{t}^{k j}\right) & =\frac{\pi_{t}^{i k} Z_{t}^{i}}{Z_{t}^{k}} \frac{\pi_{t}^{k j} Z_{t}^{k}}{Z_{t}^{j}} \\
& \leq \frac{\pi_{t}^{i j} Z_{t}^{i}}{Z_{t}^{j}}=1+\lambda_{t}^{i j}
\end{aligned}
$$

The remaining assertions follow from the discussion after Definition 1.5.

The paper is organized as follows.

In section 2, we state and prove the "abstract version" of Theorem 1.7: under condition $\left(\mathrm{NA}^{\mathrm{r}}\right)$ the cone $\widehat{A}_{T}$ of "hedgeable" or "super-replicable" claims is closed with respect to convergence in measure. This is an extension of a result from [S 92], which is sometimes refered to as Schachermayer's lemma. From this result Theorem 1.7 is deduced by standard arguments.

In section 3 we present the counter-example showing that the strict no arbitrage condition $\left(\mathrm{NA}^{\mathrm{s}}\right)$ is not suficiently strong to allow for the existence of a (not necessarily strict) consistent pricing system. The crux is, that this condition does not imply that $\widehat{A}_{T}$ is closed with respect to convergence in measure.

In section 4 we deduce from Theorem 1.7 a super-replication theorem characterizing the initial endowments $v \in \mathbb{R}^{d}$, which are portfolios in the $d$ assets, such that - starting with wealth $v$ - a given claim $\vartheta \in L^{0}\left(\Omega, \mathcal{F}_{t}, \mathbf{P}, \mathbb{R}^{d}\right)$, contingent on the $d$ assets, may be super-replicated. Under the robust no arbitrage condition $\left(\mathrm{NA}^{\mathrm{r}}\right)$, the theorem is stated and proved in full generality, without boundedness or integrability assumptions on $\vartheta$, thus extending Theorem 4.2 of [KRS 01].

Finally, in the appendix, we assemble some - mainly measure-theoretic - auxiliary results.

\section{Proof of the Theorem}

We start by formulating the "abstract version" of Theorem 1.7. It is an extension of the "crucial lemma" from [S 92] to the present setting (compare also [St 97] for a nice proof of this lemma in the frictionless setting).

Theorem 2.1 If the bid-ask process $\left(\Pi_{t}\right)_{t=0}^{T}$ has the robust no arbitrage property $\left(\mathrm{NA}^{\mathrm{r}}\right)$, then the convex cone $\widehat{A}_{T}$ is closed in $L^{0}\left(\Omega, \mathcal{F}_{T}, \mathbf{P} ; \mathbb{R}^{d}\right)$.

The fact, that Theorem 2.1 implies Theorem 1.7 is essentially standard and goes back to the work of Stricker [St 90]. In the present setting we follow the lines of [KRS 01]. The 
argument consists in the combination of a Hahn-Banach and an exhaustion argument, as is usually done in the proof of the Kreps-Yan theorem ([Y 80], [K 81]; compare also [S 92], or the survey paper [S 01]).

\section{Proof of Theorem 1.7 (admitting Theorem 2.1)}

$\left(\mathrm{NA}^{\mathrm{r}}\right) \Rightarrow(\mathrm{SCP})$ : Supposing that $\left(\Pi_{t}\right)_{t=0}^{T}$ has the robust no arbitrage property, we have to find a strictly consistent price process $\left(Z_{t}\right)_{t=0}^{T}$. We shall show that, more generally, for any given strictly positive $\mathcal{F}_{T}$-measurable function $\left.\left.\varphi: \Omega \rightarrow\right] 0,1\right]$ we may find a strictly consistent price system $Z$ in such a way that

$$
Z_{T} \leq M \varphi, \text { a.s. }
$$

for some constant $M>0$.

The proof of this feature is, in fact, standard and analogous to the usual argument showing that in the Dalang-Morton-Willinger Theorem [DMW 90] one may choose the equivalent martingale measure $\mathbf{Q}$ such that $\frac{d \mathbf{Q}}{d \mathbf{P}}$ is bounded.

We start the proof by observing that there is a bid-ask process $\left(\widetilde{\Pi}_{t}\right)_{t=0}^{T}$ with smaller bidask spreads than $\left(\Pi_{t}\right)_{t=0}^{T}$, such that $\left(\widetilde{\Pi}_{t}\right)_{t=0}^{T}$ still satisfies the robust no arbitrage condition. Indeed, by hypothesis there is a bid-ask process $\left(\bar{\Pi}_{t}\right)_{t=0}^{T}$ with smaller bid-ask spreads than $\left(\Pi_{t}\right)_{t=0}^{T}$, such that $\left(\bar{\Pi}_{t}\right)_{t=0}^{T}$ satisfies the no arbitrage condition. By Proposition A.5 below it suffices to choose $\left(\widetilde{\Pi}_{t}\right)_{t=0}^{T}$ as a bid-ask process, which is "sandwiched" between $\left(\Pi_{t}\right)_{t=0}^{T}$ and $\left(\bar{\Pi}_{t}\right)_{t=0}^{T}$ : the bid-ask process $\left(\widetilde{\Pi}_{t}\right)_{t=0}^{T}$ should have smaller bid-ask spreads than $\left(\Pi_{t}\right)_{t=0}^{T}$, while $\left(\bar{\Pi}_{t}\right)_{t=0}^{T}$ should have smaller bid-ask spreads than $\left(\widetilde{\Pi}_{t}\right)_{t=0}^{T}$. Such a choice is obviously possible (compare the proof of Proposition A.5 below).

Hence by Theorem 2.1 the convex cone $\widetilde{A}_{T}$ of hedgeable portfolios under $\left(\widetilde{\Pi}_{t}\right)_{t=0}^{T}$ is closed in $L^{0}\left(\Omega, \mathcal{F}_{T}, \mathbf{P} ; \mathbb{R}^{d}\right)$, contains $L^{0}\left(\Omega, \mathcal{F}_{T}, \mathbf{P} ; \mathbb{R}_{-}^{d}\right)$, and satisfies $\widetilde{A}_{T} \cap$ $L^{0}\left(\Omega, \mathcal{F}_{T}, \mathbf{P} ; \mathbb{R}_{+}^{d}\right)=\{0\}$.

Fix $\varphi$ as above and define the Banach space $L_{\varphi}^{1}\left(\Omega, \mathcal{F}_{T}, \mathbf{P} ; \mathbb{R}^{d}\right)$, consisting of all $f \in$ $L^{0}\left(\Omega, \mathcal{F}_{T}, \mathbf{P} ; \mathbb{R}_{+}^{d}\right)$, such that

$$
\|f\|_{L_{\varphi}^{1}}=\mathbf{E}_{\mathbf{P}}\left[\varphi(\omega)\|f(\omega)\|_{\mathbb{R}^{d}}\right]<\infty
$$

Its dual with respect to the scalar product $\langle f, g\rangle=\mathbf{E}[(f, g)]$ is the Banach space $L_{\psi}^{\infty}\left(\Omega, \mathcal{F}_{T}, \mathbf{P} ; \mathbb{R}^{d}\right)$ of $\mathcal{F}_{T}$-measurable functions $g$, such that

$$
\|g\|_{L_{\psi}^{\infty}}=\operatorname{ess} \sup _{\omega \in \Omega} \psi(\omega)\|g(\omega)\|_{\mathbb{R}^{d}}<\infty, \quad \text { where } \psi=\varphi^{-1} .
$$

The intersection of $\widetilde{A}_{T}$ with $L_{\varphi}^{1}\left(\Omega, \mathcal{F}_{T}, \mathbf{P} ; \mathbb{R}^{d}\right)$ is closed in $L_{\varphi}^{1}\left(\Omega, \mathcal{F}_{T}, \mathbf{P} ; \mathbb{R}^{d}\right)$ and its intersection with $L_{\varphi}^{1}\left(\Omega, \mathcal{F}_{T}, \mathbf{P} ; \mathbb{R}_{+}^{d}\right)$ is reduced to zero. Hence for each $D \in \mathcal{F}_{T}, \mathbf{P}[D]>0$, we may find a linear functional $g_{D} \in L_{\psi}^{\infty}\left(\Omega, \mathcal{F}_{T}, \mathbf{P} ; \mathbb{R}^{d}\right)$, which strictly separates $\left(e^{1}+\ldots+\right.$ $\left.e^{d}\right) \chi_{D}$ from $\widetilde{A}_{T}$, in the sense that $\left.G\right|_{\widetilde{A}_{T} \cap L_{\varphi}^{1}\left(\Omega, \mathcal{F}_{T}, \mathbf{P} ; \mathbb{R}^{d}\right)} \leq 0$ while $\left\langle\left(e^{1}+\ldots+e^{d}\right) \chi_{D}, g_{D}\right\rangle>0$. One readily verifies that $g_{D}$ takes its values in $\mathbb{R}_{+}^{d}$ a.s., and, using the above mentioned exhaustion argument (see, e.g. [S 92]), we may find a sequence $\left(D_{n}\right)_{n=1}^{\infty}$ in $\mathcal{F}_{T}$, and a sequence of positive scalars $a_{n}$, such that $g=\sum_{n=1}^{\infty} a_{n} g_{D_{n}}$ has the following properties:

(i) $\|g\|_{L_{\psi}^{\infty}\left(\Omega, \mathcal{F}_{T}, \mathbf{P} ; \mathbb{R}^{d}\right)}<\infty$,

(ii) $g(\omega) \in \mathbb{R}_{+}^{d} \backslash\{0\}$, a.s., 
(iii) $\langle f, g\rangle \leq 0$, for $f \in \widetilde{A}_{T} \cap L_{\varphi}^{1}\left(\Omega, \mathcal{F}_{T}, \mathbf{P} ; \mathbb{R}^{d}\right)$.

Define $Z_{T}=g$ and $Z_{t}=\mathbf{E}\left[Z_{T} \mid \mathcal{F}_{t}\right]$, to find an $\mathbb{R}^{d}$-valued martingale satisfying (20) and taking its values a.s. in $\mathbb{R}_{+}^{d} \backslash\{0\}$. We claim that $Z_{t}(\omega) \in \widetilde{K}_{t}^{*}(\omega)$ a.s., for each $t=0, \ldots, T$, where $\widetilde{K}_{t}^{*}(\omega)$ denotes the cone of consistent price systems for $\widetilde{\Pi}_{t}(\omega)$. Indeed, suppose that there is $0 \leq t \leq T$ and a set $D \in \mathcal{F}_{t}, \mathbf{P}[D>0]$, sucht that $Z_{t}(\omega) \notin \widetilde{K}_{t}^{*}(\omega)$, for $\omega \in D$. Using measurable selection we may find a bounded $\mathcal{F}_{t}$-measurable function $f$, taking its values in $-\widehat{K}_{t}(\omega)$ a.s., $f(\omega)=0$, for $\omega \notin D$, and

$$
\left(f(\omega), Z_{t}(\omega)\right)>0 \quad \text { a.s., for } \omega \in D .
$$

As $f \in \widetilde{A}_{T}$, we find a contradiction to (iii), as $\mathbf{E}\left[\left(f, Z_{t}\right)\right]=\mathbf{E}\left[\left(f, \mathbf{E}\left[Z_{T} \mid \mathcal{F}_{t}\right]\right)\right]=$ $\mathbf{E}[(f, g)] \leq 0$.

Finally, we apply Proposition A.5, to deduce that $\left(Z_{t}(\omega)\right)_{t=0}^{T}$ takes its values a.s. in the relative interior of the cones $\left(\widehat{K}_{t}^{*}(\omega)\right)_{t=0}^{T}$ of consistent price systems for $\left(\Pi_{t}(\omega)\right)_{t=0}^{T}$. This finishes the proof of the implication $\left(\mathrm{NA}^{\mathrm{r}}\right) \Rightarrow(\mathrm{SCP})$.

$(\mathrm{SCP}) \Rightarrow\left(\mathrm{NA}^{\mathrm{r}}\right)$ : Now suppose that there is a strictly consistent price system $\left(Z_{t}\right)_{t=0}^{T}$ for $\left(\Pi_{t}\right)_{t=0}^{T}$. We have to find a bid-ask process $\left(\widetilde{\Pi}_{t}\right)_{t=0}^{T}$ with smaller bid-ask spreads than $\left(\Pi_{t}\right)_{t=0}^{T}$, satisfying $(\mathrm{NA})$.

To do so, we define

$$
\tilde{\pi}_{t}^{i j}(\omega)=\frac{Z_{t}^{j}(\omega)}{Z_{t}^{i}(\omega)} .
$$

The resulting bid-ask matrices $\widetilde{\Pi}_{t}(\omega)$ are frictionless, so that their "bid-ask spreads" are reduced to the singletons $\left\{\tilde{\pi}_{t}^{i j}(\omega)\right\}$, which are contained in the relative interior of the bid-ask spreads $\left[\frac{1}{\pi^{j i}}, \pi^{i j}\right]$.

Next we shall show that the bid-ask process $\left(\widetilde{\Pi}_{t}(\omega)\right)_{t=0}^{T}$ satisfies the no arbitrage condition (NA). Indeed, for every $f=\left(f^{1}, \ldots, f^{d}\right) \in \widetilde{A}_{T}$ such that its negative part $f_{-}:=\left(f_{-}^{1}, \ldots, f_{-}^{d}\right)$ is uniformly bounded, we have

$$
\mathbf{E}\left[\left(f, Z_{T}\right)\right] \leq 0
$$

This inequality was obtained in [KRS 01, Lemma 4]. Admitting (25) we conclude that an element $f \in \widetilde{A}_{T}$, which takes its values in $\mathbb{R}_{+}^{d}$, satisfies $f \equiv 0$, as $Z_{T}$ takes its values in the interior of $\mathbb{R}_{+}^{d}$. Hence $\left(\widetilde{\Pi}_{t}\right)_{t=0}^{T}$ satisfies (NA).

The proof of (25) is slightly subtle, because of integrability problems. Firstly remark, that, by the lower boundedness of $f$ and the boundedness of $Z_{T}$, it will suffice to show that there exists a sequence $\left(D_{n}\right)_{n=1}^{\infty}$ in $\mathcal{F}_{T}$ such that $\lim _{n \rightarrow \infty} \mathbf{P}\left[D_{n}\right]=1$ and

$$
\liminf _{n \rightarrow \infty} \mathbf{E}\left[\left(f, Z_{T}\right) \chi_{D_{n}}\right] \leq 0 .
$$

To show (26), associate to $f$ a self-financing (w.r. to $\left.\left(\widetilde{\Pi}_{t}\right)_{t=0}^{T}\right)$ portfolio process $\left(\vartheta_{0}, \ldots, \vartheta_{T}\right)$ with $\vartheta_{T}=f$, and let $\xi_{t}=\vartheta_{t}-\vartheta_{t-1}$.

Fix $n \in \mathbf{N}$, and do the following inductive construction. Let $D_{0, n}=\Omega$ and suppose that, for $1 \leq t \leq T$, the set $D_{t-1, n} \in \mathcal{F}_{t-1}$ has been defined, such that $\xi_{0}, \ldots, \xi_{t-1}$ are uniformly bounded on $D_{t-1, n}$, that $\mathbf{P}\left[D_{t-1, n}\right]>1-\frac{1}{n}$, and that $\mathbf{E}\left[\left(\left(\xi_{0}+\ldots+\xi_{t-1}\right), Z_{T}\right) \chi_{D_{t-1, n}}\right]<\frac{1}{n}$. For the inductive step, it suffices to choose $D_{t, n} \in \mathcal{F}_{t}$ 
contained in $D_{t-1, n}$, such that $\mathbf{P}\left[D_{t, n}\right]>1-\frac{1}{n}, \xi_{t}$ is uniformly bounded on $D_{t, n}$ and $\mathbf{E}\left[\left(\left(\xi_{0}+\ldots+\xi_{t-1}\right), Z_{T}\right) \chi_{D_{t, n}}\right]<\frac{1}{n}$. Note that $\mathbf{E}\left[\left(\xi_{t}, Z_{T}\right) \chi_{D_{t, n}}\right] \leq 0$, which readily shows that $D_{t, n}$ satisfies the inductive hypothesis. After finitely many steps we arrive at $D_{n}:=D_{T, n}$ such that $\mathbf{E}\left[\left(f, Z_{T}\right) \mathbf{1}_{D_{n}}\right]<\frac{1}{n}$, which proves (26) and (25).

Summing up, we have shown the implication $(\mathrm{SCP}) \Rightarrow\left(\mathrm{NA}^{\mathrm{r}}\right)$.

Remark 2.2 It is worth noting that we have shown an additional feature in the above proof of Theorem 1.7. Indeed, for any given $\mathcal{F}_{T}$-measurable function $\left.\left.\varphi: \Omega \mapsto\right] 0,1\right]$ the consistent price process $\left(Z_{t}\right)_{t=0}^{T}$ may be choosen such that $(20)$ is satisfied. For example, taking $\varphi \equiv 1$, we may choose the process to be uniformly bounded. In section 4 we shall also consider less trivial choices for $\varphi$.

We also remark for later use that, in the course of the above proof, we have shown the following result:

Lemma 2.3 Suppose that the bid-ask process $\left(\Pi_{t}\right)_{t=0}^{T}$ satisfies the robust no arbitrage condition $\left(N A^{r}\right)$, and let $\left.\left.\varphi: \Omega \rightarrow\right] 0,1\right]$ and $\psi: \Omega \rightarrow[1, \infty[$ be as above. Suppose that $g \in L_{\psi}^{\infty}\left(\Omega, \mathcal{F}_{T}, \mathbf{P} ; \mathbb{R}^{d} \backslash\{0\}\right)$ satisfies $\left.g\right|_{\widehat{A}_{T} \cap L_{\varphi}^{1}\left(\Omega, \mathcal{F}_{T}, \mathbf{P} ; \mathbb{R}^{d}\right)} \leq 0$.

Defining $Z_{t}=\mathbf{E}\left[g \mid \mathcal{F}_{t}\right]$, for $t=0, \ldots, T$, we then have that $\left(Z_{t}\right)_{t=0}^{T}$ is a consistent price process for $\left(\Pi_{t}\right)_{t=0}^{T}$.

Remark 2.4 One more remark for later use: we have shown in the last part of the proof of Theorem 1.7 that, for $\left(\Pi_{t}\right)_{t=0}^{T}$ satisfying $\left(\mathrm{NA}^{\mathrm{r}}\right), f \in \widehat{A}_{T}$, and a consistent price process

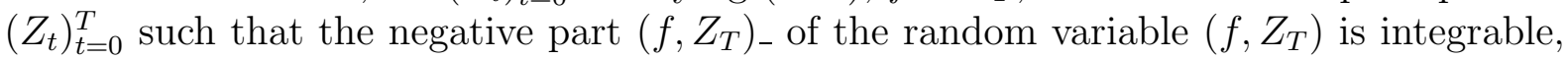
we have

$$
\mathbf{E}\left[\left(f, Z_{T}\right)\right] \leq 0,
$$

which implies in particular that the positive part $\left(f, Z_{T}\right)_{+}$is integrable too.

The rest of the section is devoted to the proof of Theorem 2.1.

We fix the $d \times d$ bid-ask process $\left(\Pi_{t}\right)_{t=0}^{T}$, based on and adapted to $\left(\Omega,\left(\mathcal{F}_{t}\right)_{t=0}^{T}, \mathbf{P}\right)$, and denote by $\mathcal{A}$ the set of all self-financing processes $\left(\vartheta_{t}\right)_{t=0}^{T}$, considered as a subset of the topological vector space $\mathcal{E}=\mathbb{R}^{d} \times L^{0}\left(\Omega, \mathcal{F}_{1}, \mathbf{P} ; \mathbb{R}^{d}\right) \times \ldots \times L^{0}\left(\Omega, \mathcal{F}_{T}, \mathbf{P} ; \mathbb{R}^{d}\right)$. Note that $\widehat{A}_{t}$ equals the projection of $\mathcal{A}$ onto the $t^{\prime}$ th coordinate of $\mathcal{E}$. By $\mathcal{N}$ we denote the subset of $\mathcal{E}$ formed by the increments $\left(\xi_{t}\right)_{t=0}^{T}=\left(\vartheta_{t}-\vartheta_{t-1}\right)_{t=0}^{T}$ of those self-financing processes $\left(\vartheta_{t}\right)_{t=0}^{T}$ such that $\vartheta_{T} \equiv 0$.

Lemma 2.5 Let $\Pi$ and $\widetilde{\Pi}$ be bid-ask matrices such that $\widetilde{\Pi}$ has smaller bid-ask spreads than $\Pi$. Let $v \in \widehat{K}(\Pi) \backslash \widehat{F}(\Pi)$, where $\widehat{K}(\Pi)$ denots the solvency cone $\widehat{K}(\Pi)$, and $\widehat{F}(\Pi)$ the space of portfolios exchangeable to zero associated to $\Pi$.

Then $v$ is in the interior of the solvency cone $\widehat{K}(\widetilde{\Pi})$. Hence there is $\varepsilon>0$, such that $v-\varepsilon \mathbf{1} \in \widehat{K}(\widetilde{\Pi})$, where $\mathbf{1}=\left(e^{1}+\ldots+e^{d}\right) \in \mathbb{R}^{d}$.

Proof By the definition of $\widehat{K}(\Pi)$, there are non-negative scalars $a^{i j}$ and $b^{i}$, such that

$$
v=\sum_{i, j} a^{i j}\left(\pi^{i j} e^{i}-e^{j}\right)+\sum_{i} b^{i} e^{i}
$$


If $b^{i} \neq 0$, for some $1 \leq i \leq d$, then $v$ is in the interior of $\widehat{K}(\Pi)$. Hence we may suppose that each $b^{i}$ equal zero.

If, for all pairs $i \neq j$ such that $a^{i j}>0$, we have $\pi^{i j} \pi^{j i}=1$, then $v \in \widehat{F}(\Pi)$, which we excluded by hypothesis. Therefore there is some $i_{0}, j_{0}$ such that $a^{i_{0} j_{0}}>0$ and $\widetilde{\pi}^{i_{0} j_{0}}<\pi^{i_{0} j_{0}}$. Hence

$$
\widetilde{v}=\sum_{i, j} a^{i j}\left(\widetilde{\pi}^{i j} e^{i}-e^{j}\right)
$$

is an element of $\widehat{K}(\widetilde{\Pi})$ such that

$$
v-\tilde{v} \geq a^{i_{0} j_{0}}\left(\pi^{i_{0} j_{0}}-\tilde{\pi}^{i_{0} j_{0}}\right) e^{i_{0}}
$$

where $\geq$ denotes the natural order, and $e^{i_{0}}$ the $i_{0}$-th unit vector in $\mathbb{R}^{d}$. As $\widehat{K}(\widetilde{\Pi})$ contains $\mathbb{R}_{+}^{d} \backslash\{0\}$ in its interior, this implies that $v$ is in the interior of $\widehat{K}(\widetilde{\Pi})$.

The final assertion of the lemma is obvious.

The subsequent lemma will be crucial for the proof of Theorem 2.1.

Lemma 2.6 If $\left(\Pi_{t}\right)_{t=0}^{T}$ satisfies the robust no arbitrage condition $\left(N A^{r}\right)$, the set $\mathcal{N}$ is a closed vector subspace of $\mathcal{E}=\mathbb{R}^{d} \times L^{0}\left(\Omega, \mathcal{F}_{1}, \mathbf{P} ; \mathbb{R}^{d}\right) \times \ldots \times L^{0}\left(\Omega, \mathcal{F}_{T}, \mathbf{P} ; \mathbb{R}^{d}\right)$.

Proof Fix $\left(\xi_{t}\right)_{t=0}^{T} \in \mathcal{N}$. By definition we have $\xi_{t}(\omega) \in-\widehat{K}_{t}(\omega)$ a.s., for $t=0, \ldots, T$. We claim that, in fact, $\xi_{t}(\omega) \in \widehat{F}_{t}(\omega)$ a.s., for $t=0, \ldots, T$. Indeed, let $\tau$ equal the stopping time

$$
\tau=\min \left\{t:-\xi_{t} \in \widehat{K}_{t} \backslash \widehat{F}_{t}\right\}
$$

and assume that the event $\{\tau<\infty\}$ has strictly positive measure.

Let $\left(\widetilde{\Pi}_{t}\right)_{t=0}^{T}$ be a bid-ask process with smaller bid-ask spreads than $\left(\Pi_{t}\right)_{t=0}^{T}$, satisfying $(N A)$, and define the portfolio process $\left(\widetilde{\vartheta}_{t}\right)_{t=0}^{T}$ with increments $\widetilde{\xi}_{t}=\widetilde{\vartheta}_{t}-\widetilde{\vartheta}_{t-1}$ in the following way

$$
\widetilde{\xi}_{t}=\left\{\begin{array}{cc}
\xi_{t} & \text { for } \quad t \neq \tau \\
\xi_{t}+\varepsilon_{t} \mathbf{1} & \text { for } \quad t=\tau
\end{array}\right.
$$

where $\varepsilon_{t}=\varepsilon_{t}(\omega)$ is the largest number such that $\xi_{t}(\omega)+\varepsilon_{t}(\omega) \mathbf{1} \in-\widehat{K}\left(\widetilde{\Pi}_{t}(\omega)\right)$. It follows from Lemma 2.5 that $\varepsilon_{t}(\omega)>0$ on $\{\tau=t\}$. Obviously, for $t=0, \ldots, T$, the map $\omega \mapsto \varepsilon_{t}(\omega)$ is well defined, finitely valued and $\mathcal{F}_{t}$-measurable.

Hence $\left(\widetilde{\vartheta}_{t}\right)_{t=0}^{T}=\left(\widetilde{\xi}_{0}+\ldots+\widetilde{\xi}_{t}\right)_{t=0}^{T}$ is a self-financing portfolio process with respect to $\left(\widetilde{\Pi}_{t}\right)_{t=0}^{T}$, yielding an arbitrage as $\widetilde{\vartheta}_{T} \geq \sum_{t=0}^{T} \varepsilon_{t} \mathbf{1} \chi_{\{\tau=t\}}$. Hence, each $\left(\widetilde{\Pi}_{t}\right)_{t=0}^{T}$ with smaller bid-ask spreads than $\left(\Pi_{t}\right)_{t=0}^{T}$ violates $(\mathrm{NA})$, a contradiction to the robust no arbitrage assumption.

For portfolio processes $\vartheta^{\prime}, \vartheta^{\prime \prime} \in \mathcal{N}$ and scalars $\lambda^{\prime}, \lambda^{\prime \prime}$, we therefore have $\lambda^{\prime} \vartheta^{\prime}+\lambda^{\prime \prime} \vartheta^{\prime \prime} \in \mathcal{N}$, i.e., $\mathcal{N}$ is a vector space. The fact, that this space is closed in $\mathcal{E}$, now follows from the fact, that each $\widehat{F}_{t}(\omega)$ is a closed subspace of $\mathbb{R}^{d}$.

In the recent paper [KRS 02], which was written on the basis of a preliminary version of the present paper, these authors point out that - once the crucial fact that $\mathcal{N}$ is a vector space is established - the remaining part of the proof of Theorem 2.1 may be quickly deduced from the results in [KRS 01] (which, in turn, has clear priority with respect to the present paper). 
We shall presently complete the proof of Theorem 1.7 in a self-contained way by following, which we briefly recall: we say that two self-financing portfolio processes $\vartheta^{1}, \vartheta^{2} \in \mathcal{A}$ are equivalent, if $\left(\xi_{t}\right)_{t=0}^{T}:=\left(\xi_{t}^{1}-\xi_{t}^{2}\right)_{t=0}^{T}=\left(\left(\vartheta_{t}^{1}-\vartheta_{t-1}^{1}\right)-\left(\vartheta_{t}^{2}-\vartheta_{t-1}^{2}\right)\right)_{t=0}^{T}$ is in $\mathcal{N}$. The preceding lemma implies that this terminology is justified, i.e., that this is indeed an equivalence relation as $\mathcal{N}$ is a vector space. Hence we may define the quotient space of $\mathcal{A}$ with respect to this equivalence relation. We may identify it with some properly chosen convex cone $\mathcal{N}^{\perp} \subseteq \mathcal{A}$ of representants of these equivalence classes, which are in some sense "orthogonal to $\mathcal{N}$ ". This allows us to select, for each $\vartheta_{T} \in \widehat{A}_{T}$, a unique process $\left(\vartheta_{0}, \ldots, \vartheta_{T}\right) \in \mathcal{N}^{\perp}$.

This selection will turn out to be an isomorphism with respect to the topology of convergence in measure, which will eventually imply that $\widehat{A}_{T}$ is closed in $L^{0}\left(\Omega, \mathcal{F}_{T}, \mathbf{P} ; \mathbb{R}^{d}\right)$, which is the assertion of Theorem 2.1 .

To be more concrete, we first show the proof of Theorem 2.1 in the (easy) one-period case $T=1$, so that the reader can get a clearer pricture - in a less abstract wording of what we have in mind.

Let $\left(\Pi_{t}\right)_{t=0}^{1}$ be a bid-ask process adapted to $\left(\Omega,\left(\mathcal{F}_{t}\right)_{t=0}^{1}, \mathbf{P}\right)$. Recall that we assumed $\mathcal{F}_{0}$ to be trivial. Let $\left(\vartheta_{0}^{n}, \vartheta_{1}^{n}\right)_{n=1}^{\infty}$ be a sequence of self-financing portfolio processes such that $\vartheta_{1}^{n}(\omega)$ converges a.s. to a random variable $\vartheta_{1}(\omega)$. We want to show that there is some $\vartheta_{0} \in \mathbb{R}^{d}$ such that $\left(\vartheta_{0}, \vartheta_{1}\right)$ is self-financing.

If $\left(\vartheta_{0}^{n}\right)_{n=1}^{\infty}$ converges in $\mathbb{R}^{d}$, then we clearly may choose $\vartheta_{0}$ to be this limit. The crux is that, in general, there is no reason why $\left(\vartheta_{0}^{n}\right)_{n=1}^{\infty}$ should converge, or even be bounded. One way to circumvent this difficulty goes as follows: denote by $\mathcal{N}_{0}$ the subset of $\mathbb{R}^{d}$ which is the projection of $\mathcal{N} \subseteq \mathbb{R}^{d} \times L^{0}\left(\Omega, \mathcal{F}_{1}, \mathbf{P} ; \mathbb{R}^{d}\right)$ onto the first coordinate. In other words, $\mathcal{N}_{0}$ consists of the vectors $\xi \in \mathbb{R}^{d}$, such that $\left(\vartheta_{1}, \vartheta_{2}\right):=(\xi, 0)$ is self-financing. By Lemma 2.6 we know that $\mathcal{N}_{0}$ is a linear sub-space of $\mathbb{R}^{d}$, and we therefore may define the orthogonal projection $p_{0}$, mapping $\mathbb{R}^{d}$ onto the orthogonal complement $\mathcal{N}_{0}^{\perp}$ of $\mathcal{N}_{0}$.

Hence we may replace the original sequence $\left(\vartheta_{0}^{n}, \vartheta_{1}^{n}\right)_{n=1}^{\infty}$ by $\left(p_{0}\left(\vartheta_{0}^{n}\right), \vartheta_{1}^{n}\right)_{n=1}^{\infty}$, which still is a sequence of self-financing portfolio processes. We now claim that $\left(p_{0}\left(\vartheta_{0}^{n}\right)\right)_{n=1}^{\infty}$ is bounded in $\mathbb{R}^{d}$. Indeed, if this were not the case, we could pass to a subsequence, still denoted by $\left(p_{0}\left(\vartheta_{0}^{n}\right)\right)_{n=1}^{\infty}$ such that $\lim _{n \rightarrow \infty}\left\|p_{0}\left(\vartheta_{0}^{n}\right)\right\|=\infty$. Define $\left(\eta_{0}^{n}, \eta_{1}^{n}\right)_{n=1}^{\infty}:=\left(\frac{p_{0}\left(\vartheta_{0}^{n}\right)}{\left\|p_{0}\left(\vartheta_{0}^{n}\right)\right\|}, \frac{\vartheta_{1}^{n}}{\left\|p_{0}\left(\vartheta_{0}^{n}\right)\right\|}\right)_{n=1}^{\infty}$, to obtain a sequence of self-financing portfolio processes, such that $\left(\eta_{1}^{n}\right)_{n=1}^{\infty}$ converges a.s. to zero. By passing again to a subsequence, we may assume that $\left(\eta_{0}^{n}\right)_{n=1}^{\infty}$ converges to some $\eta_{0}$ in the unit sphere of $\mathbb{R}^{d}$, which readily implies that $\left(\eta_{0}, 0\right)$ is a self-financing strategy. Hence $\eta_{0} \in \mathcal{N}_{0}$. On the other hand, each $\eta_{0}^{n}$ and therefore also $\eta_{0}$ is in $\mathcal{N}_{0}^{\perp}$, which yields a contradiction, showing that $\left(p_{0}\left(\vartheta_{0}^{n}\right)\right)_{n=1}^{\infty}$ is bounded. By passing once more to a subsequence, we may find a limit $\vartheta_{0} \in \mathbb{R}^{d}$ of this sequence, and $\left(\vartheta_{0}, \vartheta_{1}\right)$ then is the desired self-financing portfolio process.

The above argument shows the idea of the proof of Theorem 2.1 (which was already used in [S 92]). To extend the above argument from the case $T=1$ to the case $T \geq 2$, we only have to add some - admittedly cumbersome - technical machinery, but no additional conceptual idea.

We now pass to the proof of Theorem 2.1 in the general case: for $0 \leq t \leq T$, we denote by $\mathcal{N}_{t}$ the subset of elements $\xi_{t}=\vartheta_{t}$ in $L^{0}\left(\Omega, \mathcal{F}_{t}, \mathbf{P} ; \mathbb{R}^{d}\right)$, such that $\vartheta_{t}$ may be extended into a self-financing portfolio of the form $\left(0, \ldots, 0, \vartheta_{t}, \vartheta_{t+1}, \ldots, \vartheta_{T-1}, 0\right)$. By the preceding lemma, $\mathcal{N}_{t}$ is a linear subspace of $L^{0}\left(\Omega, \mathcal{F}_{t}, \mathbf{P} ; \mathbb{R}^{d}\right)$. The space $\mathcal{N}_{t}$ satisfies the 
assumptions of Lemma A.4, so that there is an a.s. unique $\mathcal{F}_{t}$-mesurable map $\omega \mapsto N_{t}(\omega)$ taking its values in the subspaces of $\mathbb{R}^{d}$, such that $\vartheta_{t} \in \mathcal{N}_{t}$ iff $\vartheta_{t}(\omega) \in N_{t}(\omega)$ almost surely. Obviously $N_{t}(\omega)$ is a.s. a subspace of $\widehat{F}_{t}(\omega)$.

For fixed $\omega \in \Omega$ and $0 \leq t \leq T$, denote by $p_{N_{t}(\omega)}$ the orthogonal projection, mapping $\mathbb{R}^{d}$ onto the orthogonal complement $N_{t}^{\perp}(\omega)$ of $N_{t}(\omega)$. We define the continuous, linear projection $p_{t}$ on $L^{0}\left(\Omega, \mathcal{F}_{t}, \mathbf{P} ; \mathbb{R}^{d}\right)$, by applying the projections $p_{N_{t}(\omega)}$ in a pointwise way, i.e.,

$$
p_{t}\left(\vartheta_{t}\right)(\omega)=p_{N_{t}(\omega)}\left(\vartheta_{t}(\omega)\right), \quad \text { for } \quad \vartheta_{t} \in L^{0}\left(\Omega, \mathcal{F}_{t}, \mathbf{P} ; \mathbb{R}^{d}\right) .
$$

Clearly $\operatorname{ker}\left(p_{t}\right)=\mathcal{N}_{t}$ and we write $\mathcal{N}_{t}^{\perp}$ for the subspace $\operatorname{Im}\left(p_{t}\right)$ of $L^{0}\left(\Omega, \mathcal{F}_{t}, \mathbf{P} ; \mathbb{R}^{d}\right)$, and $\mathcal{N}^{\perp}$ for the subspace $\mathcal{N}_{0}^{\perp} \times \mathcal{N}_{1}^{\perp} \times \ldots \times \mathcal{N}_{T}^{\perp}$ of $\mathcal{E}$.

For $t=0, \ldots, T$, we denote by $\mathcal{N}_{0, \ldots, t}$ the projection of $\mathcal{N}$ onto the first $t+1$ coordinates. Obviously $\mathcal{N}_{0, \ldots, t}$ consists of those elements $\left(\xi_{0}, \ldots, \xi_{t}\right) \in \mathbb{R}^{d} \times \ldots \times L^{0}\left(\Omega, \mathcal{F}_{t}, \mathbf{P} ; \mathbb{R}^{d}\right)$, for which there are increments $\left(\widetilde{\xi}_{t+1}, \ldots, \widetilde{\xi}_{T}\right)$, such that $\left(\xi_{0}, \ldots, \xi_{t}, \widetilde{\xi}_{t+1}, \ldots, \widetilde{\xi}_{T}\right) \in \mathcal{N}$.

We define $\varphi_{t}: \mathcal{N}_{0, \ldots, t-1} \rightarrow L^{0}\left(\Omega, \mathcal{F}_{t}, \mathbf{P} ; \mathbb{R}^{d}\right)$ in the following way: for $\left(\xi_{0}, \ldots, \xi_{t-1}\right) \in$ $\mathcal{N}_{0, \ldots, t-1}$ let $\left(\xi_{0}, \ldots, \xi_{t-1}, \widetilde{\xi}_{t}, \ldots, \widetilde{\xi}_{T}\right)$ be an arbitrary element in $\mathcal{N}$ extending $\left(\xi_{0}, \ldots, \xi_{t-1}\right)$, and define

$$
\varphi_{t}\left(\xi_{0}, \ldots, \xi_{t-1}\right):=p_{t}\left(\widetilde{\xi}_{t}\right) .
$$

One readily verifies that this definition is independent of the choice of the extending sequence $\left(\widetilde{\xi}_{t}, \ldots, \widetilde{\xi}_{T}\right)$ and defines a linear map $\varphi_{t}$, taking its values in $\mathcal{N}_{t}^{\perp}$.

We mention in passing, that it is not hard to show that $\varphi_{t}$, and therefore also the map $p: \mathcal{E} \rightarrow \mathcal{E}$ defined below, is continuous with respect to the topology of convergence in measure (compare the proof of Lemma A.4 below). As we shall not need this fact, we do not give the proof.

Observe that, for $\left(\xi_{0}, \ldots, \xi_{t-1}\right) \in \mathcal{N}_{0, \ldots, t-1}$, there is some extension of the form $\left(\xi_{0}, \ldots, \xi_{t-1}, \varphi_{t}\left(\xi_{0}, \ldots, \xi_{t-1}\right), \widetilde{\xi}_{t+1}, \ldots, \widetilde{\xi}_{T}\right) \in \mathcal{N}$. Also note, that for $\left(\xi_{0}, \ldots, \xi_{T-1}\right) \in$ $\mathcal{N}_{0, \ldots, T-1}$ we have $\varphi_{T}\left(\xi_{0}, \ldots, \xi_{T-1}\right)=-\left(\xi_{0}+\ldots+\xi_{T-1}\right)$.

Finally we define the map $p$ from $\mathcal{E}=\mathbb{R}^{d} \times \ldots \times L^{0}\left(\Omega, \mathcal{F}_{T}, \mathbf{P} ; \mathbb{R}^{d}\right)$ onto itself by defining inductively the elements $\left(\bar{\xi}_{0}, \ldots, \bar{\xi}_{T}\right)=p\left(\xi_{0}, \ldots, \xi_{T}\right)$ :

$$
\begin{aligned}
\bar{\xi}_{0} & =p_{0}\left(\xi_{0}\right), \\
\bar{\xi}_{1} & =p_{1}\left(\xi_{1}\right)-\varphi_{1}\left(\xi_{0}-\bar{\xi}_{0}\right), \\
& \vdots \\
\bar{\xi}_{t} & =p_{t}\left(\xi_{t}\right)-\varphi_{t}\left(\left(\xi_{0}, \ldots, \xi_{t-1}\right)-\left(\bar{\xi}_{0}, \ldots, \bar{\xi}_{t-1}\right)\right), \\
& \vdots \\
\bar{\xi}_{T} & =p_{T}\left(\xi_{T}\right)-\varphi_{T}\left(\left(\xi_{0}, \ldots, \xi_{T-1}\right)-\left(\bar{\xi}_{0}, \ldots, \bar{\xi}_{T-1}\right)\right) \\
& =\xi_{T}+\left(\xi_{0}+\ldots+\xi_{T-1}\right)-\left(\bar{\xi}_{0}+\ldots+\bar{\xi}_{T-1}\right) .
\end{aligned}
$$

Lemma 2.7 The map $p: \mathcal{E} \rightarrow \mathcal{E}$ is a linear map with $\operatorname{ker}(p)=\mathcal{N}$ and $\operatorname{Im}(p)=\mathcal{N}^{\perp}=$ $\mathcal{N}_{0}^{\perp} \times \mathcal{N}_{1}^{\perp} \times \ldots \times \mathcal{N}_{T}^{\perp}$.

Proof The fact, that the map $p$ is linear, follows from the fact that we have written each of its coordinates as the composition of linear maps. 
To see that $\mathcal{N} \subseteq \operatorname{ker}(p)$, fix $\left(\xi_{0}, \ldots, \xi_{T}\right) \in \mathcal{N}$, and let us verify inductively that $\bar{\xi}_{0}=$ $0, \bar{\xi}_{1}=0, \ldots, \bar{\xi}_{T}=0$. This is obvious for $\bar{\xi}_{0}$. Suppose we know that $\bar{\xi}_{0}=\ldots=\bar{\xi}_{t-1}=0$. Then on one hand side

$$
\left(\xi_{0}, \ldots, \xi_{t-1}, \xi_{t}, \ldots, \xi_{T}\right) \in \mathcal{N}
$$

On the other hand, we may find $\left(\widetilde{\xi}_{t+1}, \ldots, \widetilde{\xi}_{T}\right)$ such that

$$
\left(\xi_{0}, \ldots, \xi_{t-1}, \varphi_{t}\left(\xi_{0}, \ldots, \xi_{t-1}\right), \widetilde{\xi}_{t+1}, \ldots, \widetilde{\xi}_{T}\right) \in \mathcal{N}
$$

Hence

$$
\left(0, \ldots, 0, \xi_{t}-\varphi_{t}\left(\xi_{0}, \ldots, \xi_{t-1}\right), \xi_{t+1}-\widehat{\xi}_{t+1}, \ldots, \xi_{T}-\widehat{\xi}_{T}\right) \in \mathcal{N}
$$

and therefore

$$
\xi_{t}-\varphi_{t}\left(\xi_{0}, \ldots, \xi_{t-1}\right) \in \mathcal{N}_{t}
$$

so that

$$
p_{t}\left(\xi_{t}-\varphi_{t}\left(\xi_{0}, \ldots, \xi_{t-1}\right)\right)=p_{t}\left(\xi_{t}\right)-\varphi_{t}\left(\xi_{0}, \ldots, \xi_{t-1}\right)=0,
$$

which shows that $\bar{\xi}_{t}=0$.

Next we verify that, for $\left(\xi_{0}, \ldots, \xi_{T}\right) \in \mathcal{N}^{\perp}=\mathcal{N}_{0}^{\perp} \times \mathcal{N}_{1}^{\perp} \times \ldots \times \mathcal{N}_{T}^{\perp}$, we have $p\left(\xi_{0}, \ldots, \xi_{T}\right)=\left(\xi_{0}, \ldots, \xi_{T}\right)$. This is done by an inductive argument similar as above, and therefore skipped.

Finally observe that the vector spaces $\mathcal{N}$ and $\mathcal{N}^{\perp}$ linearly span $\mathcal{E}=\mathbb{R}^{d} \times \ldots \times$ $L^{0}\left(\Omega, \mathcal{F}_{T}, \mathbf{P} ; \mathbb{R}^{d}\right)$. Hence $p$ is a projection onto $\mathcal{N}^{\perp}$ with $\operatorname{ker}(p)=\mathcal{N}$.

Proof of Theorem 2.1 Let $\left(\vartheta_{0}^{n}, \ldots, \vartheta_{T}^{n}\right)$ be a sequence of self-financing portfolio processes such that $\vartheta_{T}^{n}$ converges to some $\vartheta_{T}$ in the topology of $L^{0}\left(\Omega, \mathcal{F}_{T}, \mathbf{P} ; \mathbb{R}^{d}\right)$, i.e., with respect to convergence in measure. We have to show that there is a self-financing portfolio process of the form $\left(\vartheta_{0}, \ldots, \vartheta_{T}\right)$.

By passing to a subsequence we may and do assume that $\left(\vartheta_{T}^{n}\right)_{n=1}^{\infty}$ a.s. converges to $\vartheta_{T}$.

Denote by $\left(\xi_{0}^{n}, \ldots, \xi_{T}^{n}\right)_{n=1}^{\infty}$ the sequence of increments $\left(\vartheta_{0}^{n}, \vartheta_{1}^{n}-\vartheta_{0}^{n}, \ldots, \vartheta_{T}^{n}-\vartheta_{T-1}^{n}\right)_{n=1}^{\infty}$ and let

$$
\left(\bar{\xi}_{0}^{n}, \ldots, \bar{\xi}_{T}^{n}\right)=p\left(\xi_{0}^{n}, \ldots, \xi_{T}^{n}\right),
$$

where the projection $p: \mathcal{E} \rightarrow \mathcal{E}$ is given by the preceding lemma. Clearly $\left(\bar{\xi}_{0}^{n}, \ldots, \bar{\xi}_{T}^{n}\right)_{n=1}^{\infty}$ is a sequence of increments of self-financing portfolio processes such that $\vartheta_{T}^{n}=\bar{\xi}_{0}^{n}+\ldots+\bar{\xi}_{T}^{n}$, for each $n \in \mathbf{N}$.

To alleviate notation we still write $\left(\xi_{0}^{n}, \ldots, \xi_{T}^{n}\right)$ for $\left(\bar{\xi}_{0}^{n}, \ldots, \bar{\xi}_{T}^{n}\right)$, i.e., we assume w.l.g. that the sequence $\left(\xi_{0}^{n}, \ldots, \xi_{T}^{n}\right)$ is in $\mathcal{N}^{\perp}$.

Claim 1 For each $t=0, \ldots, T$, we have

$$
\limsup _{n \rightarrow \infty}\left\|\xi_{t}^{n}\right\|_{\mathbb{R}^{d}}<\infty, \quad \text { a.s. }
$$

Admitting claim 1 for a moment, we can finish the proof by the following inductive argument. For $t=0$, we extract an increasing sequence $\left(\tau_{k}^{0}\right)_{k=1}^{\infty}$ such that the sequence of vectors $\left(\xi_{0}^{\tau_{k}^{0}}\right)_{k=1}^{\infty}$ in $\mathbb{R}^{d}$ converges to some $\xi_{0} \in \mathbb{R}^{d}$. We have used the strange notation $\left(\tau_{k}^{0}\right)_{k=1}^{\infty}$ to denote a (deterministic) increasing sequence $\left(n_{k}\right)_{k=1}^{\infty}=\left(\tau_{k}^{0}\right)_{k=1}^{\infty}$, as we shall 
need the more general notion of increasing random sequences (defined and discussed in the appendix) in the subsequent induction steps.

For the formulation of the induction hypothesis, fix $1 \leq t \leq T$, and suppose that there has been defined an $\mathcal{F}_{t-1}$-measurable increasing random sequence $\left(\tau_{k}^{t-1}\right)_{k=1}^{\infty}$ and an adapted process $\left(\xi_{0}, \ldots, \xi_{t-1}\right)$, such that, for $0 \leq i \leq t-1$, we have $\xi_{i}=\lim _{k \rightarrow \infty} \xi_{i}^{\tau_{k}^{t-1}}$ almost surely.

For the induction step we use (42) and Lemma A.2 to find an $\mathcal{F}_{t}$-measurable increasing random subsequence $\left(\tau_{k}^{t}\right)_{k=1}^{\infty}$ of $\left(\tau_{k}^{t-1}\right)_{k=1}^{\infty}$, such that $\left(\xi_{t}^{\tau_{k}^{t}}\right)_{k=1}^{\infty}$ converges a.s. to some $\mathcal{F}_{t^{-}}$ measurable random variable $\xi_{t}$. This completes the inductive step.

Doing the induction for $t=0, \ldots, T$, we obtain the increments $\left(\xi_{0}, \ldots, \xi_{T}\right)$ of a process $\vartheta=\left(\vartheta_{0}, \ldots, \vartheta_{T}\right)$. It is straightforward to verify that $\vartheta$ is a self-financing portfolio process such that, for its $T$-th coordinate we have $\vartheta_{T}=\lim _{n \rightarrow \infty} \vartheta_{T}^{n}$ almost surely. This finishes the proof of Theorem 2.1.

We still have to prove claim 1 . To do so we formulate a second claim.

Claim 2 Suppose that $\left(\vartheta_{0}^{n}, \ldots, \vartheta_{T}^{n}\right)_{n=1}^{\infty}$ is a sequence of self-financing processes with increments $\left(\xi_{0}^{n}, \ldots, \xi_{T}^{n}\right) \in \mathcal{N}^{\perp}$ such that $\left(\vartheta_{T}^{n}\right)_{n=1}^{\infty}$ is a.s. bounded and (42) fails.

Then there exists a sequence $\left(\eta_{0}^{n}, \ldots, \eta_{T}^{n}\right)_{n=1}^{\infty}$ of self-financing processes with increments $\left(\zeta_{0}^{n}, \ldots, \zeta_{T}^{n}\right)_{n=1}^{\infty} \in \mathcal{N}^{\perp}$ such that

(i) $\lim \sup _{n \rightarrow \infty}\left\|\zeta_{t}^{n}\right\|<\infty$ a.s., for $0 \leq t \leq T$,

(ii) $\mathbf{P}\left[\lim \sup _{n \rightarrow \infty}\left\|\zeta_{t}^{n}\right\|_{\mathbb{R}^{d}}>0\right]>0$, for some $t \in\{0, \ldots, T\}$,

(iii) $\lim _{n \rightarrow \infty} \eta_{T}^{n}=0$ a.s.

To verify Claim 2 let $t_{0}$ be the smallest $t \in\{0, \ldots, T\}$ such that (42) fails. Define $\widehat{A}_{t_{0}}=\left\{\lim \sup _{n \rightarrow \infty}\left\|\xi_{t_{0}}^{n}\right\|=\infty\right\}$ and define $\left(\zeta_{t}^{n}\right)_{t=0}^{T}$ by letting $\zeta_{t}^{n}(\omega)=0$ if either $0 \leq t<t_{0}$ or $\omega \notin \widehat{A}_{t_{0}}$. To define the remaining values of $\left(\zeta_{t}^{n}\right)_{t=0}^{T}$ we proceed as follows.

Use Lemma A.2 to find an $\mathcal{F}_{t_{0}}$-measurable increasing random sequence $\left(\tau_{n}\right)_{n=1}^{\infty}$ such that, for a.e. $\omega \in \widehat{A}_{t_{0}}$, we have $\xi_{t_{0}}^{\tau_{n}(\omega)}(\omega) \neq 0$ for all $n$, and

$$
\lim _{n \rightarrow \infty}\left\|\xi_{t_{0}}^{\tau_{n}(\omega)}(\omega)\right\|_{\mathbb{R}^{d}}=\infty
$$

Defining

$$
\zeta_{t}^{n}(\omega)=\frac{\xi_{t}^{\tau_{n}(\omega)}(\omega)}{\left\|\xi_{t_{0}}^{\tau_{n}(\omega)}(\omega)\right\|_{\mathbb{R}^{d}}}, \quad \text { for } t_{0} \leq t \leq T, \omega \in \widehat{A}_{t_{0}},
$$

we have found a sequence $\left(\zeta_{0}^{n}, \ldots, \zeta_{T}^{n}\right)_{n=1}^{\infty}$ in $\mathcal{N}^{\perp}$, such that (ii) and (iii) are satisfied and (i) holds true for $0 \leq t \leq t_{0}$.

Repeating this construction finitely many times, we finally arrive at a sequence of processes, still denoted by $\left(\zeta_{0}^{n}, \ldots, \zeta_{T}^{n}\right)_{n=1}^{\infty}$ in $\mathcal{N}^{\perp}$ satisfying (i), (ii) and (iii).

Having proved Claim 2 we shall now show that the negation of Claim 1 leads to a contradiction. 
Indeed, we then may find $\left(\zeta_{0}^{n}, \ldots, \zeta_{T}^{n}\right)_{n=1}^{\infty} \in \mathcal{N}^{\perp}$ as in Claim 2. Let $t_{0}$ be the smallest $t \in\{0, \ldots, T\}$ such that the probability in (ii) is strictly positive, and apply again Lemma A.2 to find an $\mathcal{F}_{t_{0}}$-measurable increasing random sequence $\left(\tau_{k}^{t_{0}}\right)_{k=1}^{\infty}$ such that

$$
\zeta_{t_{0}}:=\lim _{k \rightarrow \infty} \zeta_{t_{0}}^{\tau_{k}^{t_{0}}}
$$

exists and satisfies $\left\|\zeta_{t_{0}}\right\|_{\mathbb{R}^{d}}=\lim \sup _{n \rightarrow \infty}\left\|\zeta_{t_{0}}^{n}\right\|_{\mathbb{R}^{d}}$, almost surely. Choose inductively subsequences $\left(\tau_{k}^{t}\right)_{k=1}^{\infty}$, for $t_{0}<t \leq T$, similarly as after (42) above, such that

$$
\zeta_{t}:=\lim _{k \rightarrow \infty} \zeta_{t}^{\tau_{k}^{t}}, \quad \text { for } t_{0}<t \leq T
$$

exists almost surely.

Defining $\zeta_{t}(\omega)$ to equal zero for $t=0, \ldots, t_{0}-1$, we find the increments of a selffinancing process $\left(\eta_{t}\right)_{t=0}^{T}$ such that $\eta_{0}=\ldots=\eta_{t_{0}-1}=\eta_{T}=0$. Clearly $\left(\zeta_{t}\right)_{t=0}^{T} \in \mathcal{N}^{\perp}$, whence $\zeta_{t_{0}} \in \mathcal{N}_{t_{0}}^{\perp}$ almost surely. On the other hand, by the definition of $\mathcal{N}$ we have $\zeta_{t_{0}} \in \mathcal{N}_{t_{0}}$ almost surely, a contradiction to $\mathbf{P}\left[\zeta_{t_{0}} \neq 0\right]>0$, finishing the proof.

Remark 2.8 An inspection of the proof of Theorem 2.1 reveals that we only used the assumption that $\mathcal{N}$ is a linear space. Hence we have proved the following "very abstract" version of Theorem 2.1, which may be formulated without using the word arbitrage:

Let $\left(\Pi_{t}\right)_{t=0}^{T}$ be a bid ask process such that the set $\mathcal{N}$, defined in the paragraph preceding Lemma 2.7 , is a linear space. Then $\widehat{A}_{T}$ is closed in $L^{0}\left(\Omega, \mathcal{F}_{T}, \mathbf{P} ; \mathbb{R}^{d}\right)$.

It was pointed out in [KRS 02], that this latter result may also be deduced by adapting the arguments in [KRS 01], where the situation was considered, when $\mathcal{N}$ is reduced to $\{0\}$.

Remark 2.9 To finish this section let us comment on Corollary 1.10, which states that $\left(\mathrm{NA}^{\mathrm{r}}\right)$ is a sufficient condition for the decomposability of a bid-ask process $\Pi$ into an arbitrage-free price process $S$ and a transaction cost process $\Lambda$.

This result may also be formulated as a if and only if statement. To do so, we have to introduce some new notation: we call a transaction cost matrix $\Lambda$ strict if $\lambda^{i j}>0$ iff $\lambda^{j i}>0$, for each $1 \leq i, j \leq d$. A process $\Lambda=\left(\Lambda_{t}\right)_{t=0}^{T}$ taking values in the set of strict transaction cost matrices is called a strict transaction cost process.

With this terminology we may slightly sharpen Corollary 1.10: A bid-ask process $\Pi=\left(\Pi_{t}\right)_{t=0}^{T}$ satisfies the robust no arbitrage condition $\left(\mathrm{NA}^{\mathrm{r}}\right)$ if and only if there are $S=\left(S_{t}^{1}, \ldots, S_{t}^{d}\right)_{t=0}^{T}$ and $\Lambda=\left(\Lambda_{t}\right)_{t=0}^{T}$ as described in Corollary 1.10, such that $\Lambda=\left(\Lambda_{t}\right)_{t=0}^{T}$ is a strict transaction cost process.

Indeed, for the strictly consistent price system $Z$, defined in the proof of Corollary 1.10, equations (8) and (3) define a strict transaction cost process $\Lambda$.

Conversely, if the bid-ask process $\Pi$ can be decomposed into a price process $S$ and a strict transaction cost process $\Lambda$ via (16), then

$$
\tilde{\pi}_{t}^{i j}(\omega)=\frac{S_{t}^{j}(\omega)}{S_{t}^{i}(\omega)}, \quad 1 \leq i, j \leq d
$$

defines a (frictionless) bid-ask process $\widetilde{\Pi}$ satisfying (NA) with smaller bid-ask spreads than $\Pi$. 


\section{A Counter-Example}

In this section we shall show that the notions (NA) of no arbitrage and $\left(\mathrm{NA}^{\mathrm{s}}\right)$ of no arbitrage in the strict sense are not sufficiently strong to yield a version of the Fundamental Theorem of Asset Pricing in the present context.

Example 3.1 For $d=4$ and $T=1$, there is an $\mathbb{R}^{d}$-valued bid-ask process $\left(\Pi_{t}\right)_{t=0}^{1}$ satisfying (NA), i.e., verifying $\widehat{A}_{T} \cap L^{0}\left(\Omega, \mathcal{F}_{T}, \mathbf{P} ; \mathbb{R}_{+}^{d}\right)=\{0\}$, but such that

$$
\bar{A}_{T} \cap L^{0}\left(\Omega, \mathcal{F}_{T}, \mathbf{P} ; \mathbb{R}_{+}^{d}\right) \supsetneq\{0\},
$$

where $\bar{A}_{T}$ denotes the closure of $\widehat{A}_{T}$ with respect to convergence in measure.

Proof The underlying probability space $\Omega$ is chosen to be countable; we write it as $\Omega=\left\{\omega_{0}, \omega_{1}, \ldots, \omega_{n}, \ldots\right\}$. The sigma-algebra $\mathcal{F}_{0}$ is trivial, i.e., $\mathcal{F}_{0}=\{\emptyset, \Omega\}$, while $\mathcal{F}_{1}$ equals the power set of $\Omega$. For $\mathbf{P}$ we choose any probability measure on $\mathcal{F}_{1}$ assigning strictly positive weight to each $\omega \in \Omega$, e.g., $\mathbf{P}\left[\omega_{n}\right]=2^{-(n+1)}$.

We now define the bid-ask matrices $\Pi_{0}$ and $\Pi_{1}(\omega)$ : on the diagonal these $4 \times 4$ matrices all have entries equal to one, which will not further be mentioned. To define the offdiagnoal elements, fix $1>\varepsilon>0$.

$\Pi_{0}$ : For the bid-ask matrix $\Pi_{0}$, we let $\pi_{0}^{21}=\pi_{0}^{43}=1$, while $\pi_{0}^{i j}=1+\varepsilon$, for all other pairs $1 \leq i \neq j \leq 4$.

An economic interpretation goes as follows: the vector $w=(1,1,1,1)$ defines a consistent price system for $\Pi_{0}$. Fixing this price system, the exchange of one unit of asset 2 into one unit of asset 1 (and, similarly, of one unit of asset 4 into one unit of asset 3) may be done without transaction cost, while, for all other exchanges, transaction costs with a proportionality factor $\varepsilon$ have to be paid.

The solvency cone $\widehat{K}_{0}=\widehat{K}\left(\Pi_{0}\right)$ is generated by $e^{1}, e^{2}, e^{3}, e^{4},\left(e^{2}-e^{1}\right),\left(e^{4}-e^{3}\right)$ and $\left((1+\varepsilon) e^{i}-e^{j}\right)$ for the other pairs $i \neq j$.

$\Pi_{1}$ : The bid-ask matrix $\Pi_{1}$ depends on $\omega_{n} \in \Omega$.

For $n=0$ let $\pi_{1}^{i 4}\left(\omega_{0}\right)=\frac{1}{2}$ and $\pi_{1}^{4 i}\left(\omega_{0}\right)=2$, for $i=1,2,3$, and $\pi_{1}^{i j}\left(\omega_{0}\right)=1$, for $1 \leq i \neq j \leq 3$.

For $n=1$, let $\pi_{1}^{i j}\left(\omega_{1}\right)=1$, for $1 \leq i \neq j \leq 4$.

Note that $\Pi_{1}\left(\omega_{0}\right)$ and $\Pi_{1}\left(\omega_{1}\right)$ are frictionless bid-ask matrices with unique (up to a multiplicative factor) consistent price systems $\left(1,1,1, \frac{1}{2}\right)$ and $(1,1,1,1)$ respectively.

For $n \geq 2$, let $\pi_{1}^{14}\left(\omega_{n}\right)=n, \pi_{1}^{41}\left(\omega_{n}\right)=\frac{1}{n} ; \pi_{1}^{23}\left(\omega_{n}\right)=n, \pi_{1}^{32}\left(\omega_{n}\right)=\frac{1}{n} ; \pi_{1}^{43}\left(\omega_{n}\right)=1$, $\pi_{1}^{34}\left(\omega_{n}\right)=1+\varepsilon$; this well-defines $\pi_{1}^{i j}\left(\omega_{n}\right)$ for all other pairs $1 \leq i \neq j \leq d$ via

$$
\pi_{1}^{i j}\left(\omega_{n}\right)=\min \left\{\pi_{1}^{i i_{1}}\left(\omega_{n}\right) \pi_{1}^{i_{1} i_{2}}\left(\omega_{n}\right) \ldots \pi_{1}^{i_{k} j}\left(\omega_{n}\right)\right\}
$$

where the min is taken over all $k \geq 0$ and all sequences of the form $\left\{i, i_{1}, \ldots, i_{k}, j\right\}$, similarly as in (1) above.

We thus have defined the bid-ask process $\left(\Pi_{t}\right)_{t=0}^{1}$. The idea behind the above definition is best described by the construction of the following sequence $\left(\xi_{0}^{N}, \xi_{1}^{N}\right)_{N=1}^{\infty}$ of increments of self-financing portfolio processes, which might be called a "free lunch", i.e., a sequence of portfolio processes approximating an arbitrage. 
For $t=0$, define

$$
\xi_{0}^{N}=N\left(e^{1}-e^{2}\right)+\left(e^{3}-e^{4}\right) \in-\widehat{K}\left(\Pi_{0}\right) .
$$

For $t=1$ we distinguish four cases:

for $n=0$, let

$$
\xi_{1}^{N}\left(\omega_{0}\right)=-N\left(e^{1}-e^{2}\right)-\left(\frac{e^{3}}{2}-e^{4}\right) \in-\widehat{K}\left(\Pi_{1}\left(\omega_{0}\right)\right),
$$

for $n=1$, let

$$
\xi_{1}^{N}\left(\omega_{1}\right)=-N\left(e^{1}-e^{2}\right)-\left(e^{3}-e^{4}\right) \in-\widehat{K}\left(\Pi_{1}\left(\omega_{1}\right)\right),
$$

for $2 \leq n \leq N$, let

$$
\begin{aligned}
\xi_{1}^{N}\left(\omega_{n}\right) & =-\frac{N}{n}\left(n e^{1}-e^{4}\right)-N\left(\frac{e^{3}}{n}-e^{2}\right)-\left(\frac{N}{n}-1\right)\left(e^{4}-e^{3}\right) \\
& =-N\left(e^{1}-e^{2}\right)-\left(e^{3}-e^{4}\right) \in-\widehat{K}\left(\Pi_{1}\left(\omega_{n}\right)\right),
\end{aligned}
$$

and, for $n>N$, let

$$
\begin{aligned}
\xi_{1}^{N}\left(\omega_{n}\right) & =-\frac{N}{n}\left(n e^{1}-e^{4}\right)-N\left(\frac{e^{3}}{n}-e^{2}\right)-\frac{N}{n}\left(e^{4}-e^{3}\right) \\
& =-N\left(e^{1}-e^{2}\right) \in-\widehat{K}\left(\Pi_{1}\left(\omega_{n}\right)\right) .
\end{aligned}
$$

It is elementary to calculate $\vartheta_{1}^{N}=\xi_{0}^{N}+\xi_{1}^{N}$ :

$$
\vartheta_{1}^{N}\left(\omega_{n}\right)=\left\{\begin{array}{cll}
\frac{e^{3}}{2}, & \text { for } & n=0 \\
0, & \text { for } & n=1, \ldots, N \\
\left(e^{3}-e^{4}\right), & \text { for } & n>N
\end{array}\right.
$$

Hence the sequence of random variables $\left(\vartheta_{1}^{N}\right)_{N=1}^{\infty}$ converges a.s. to the random variable $\vartheta_{1}$, which equals $\vartheta_{1}\left(\omega_{0}\right)=\frac{e^{3}}{2}$, for $n=0$, and $\vartheta_{1}\left(\omega_{n}\right)=0$, for $n \geq 1$, which implies (48).

We still have to show that $\left(\Pi_{t}\right)_{t=0}^{1}$ satisfies (NA). Fix the increments $\left(\xi_{0}, \xi_{1}(\omega)\right)$ of a self-financing portfolio process $\left(\vartheta_{0}, \vartheta_{1}(\omega)\right)=\left(\xi_{0}, \xi_{0}+\xi_{1}(\omega)\right)$, such that $\vartheta_{1}(\omega) \in \mathbb{R}_{+}^{4}$, for all $\omega \in \Omega$.

As a first step, we show that $\xi_{0}$ is in the convex cone generated by $\left(e^{1}-e^{2}\right)$ and $\left(e^{3}-e^{4}\right)$. Indeed, using the hypothesis $\vartheta_{1}\left(\omega_{1}\right) \in \mathbb{R}_{+}^{4}$, we must have $\xi_{0} \in-\widehat{K}\left(\Pi_{0}\right) \cap \widehat{K}\left(\Pi_{1}\left(\omega_{1}\right)\right)$. As the linear functional $w=(1,1,1,1)$ separates these two cones, it is straightforward to verify that the intersection of these two cones equals the cone generated by $\left(e^{1}-e^{2}\right)$ and $\left(e^{3}-e^{4}\right)$.

As a second step, we show that $\xi_{0}$ is, in fact, in the cone generated by $\left(e^{1}-e^{2}\right)$. Indeed, suppose that $\xi_{0}=a\left(e^{1}-e^{2}\right)+b\left(e^{3}-e^{4}\right)$ with $a \geq 0$ and $b>0$. We claim that, for $n$ sufficiently large, $\xi_{0}$ is not in $\widehat{K}\left(\Pi_{1}\left(\omega_{n}\right)\right)$, which will give the desired contradiction. To verify this claim, observe that $w_{n}=\left(\frac{1+\varepsilon}{n}, \frac{1}{n}, 1,1+\varepsilon\right)$ is non-negative on the vectors $\left(e^{i}\right)_{i=1}^{4}$ and $\left(\pi_{1}^{i j}\left(\omega_{n}\right) e^{i}-e^{j}\right)_{1 \leq i, j \leq d}$, which generate $\widehat{K}\left(\Pi_{1}\left(\omega_{n}\right)\right)$, so that $w_{n}$ is a consistent price system for $\Pi_{1}\left(\omega_{n}\right)$. On the other hand, for $n$ large enough, we have $\left(a\left(e^{1}-e^{2}\right)+b\left(e^{3}-\right.\right.$ $\left.\left.e^{4}\right), w_{n}\right)=\frac{\varepsilon a}{n}-\varepsilon b<0$, which again gives a contradiction. 
Hence the only candidates for $\xi_{0}$ are the positive multiples of $\left(e^{1}-e^{2}\right)$. Glancing at the definitions of $\Pi_{1}(\omega)$, one readily verifies that $e^{1}-e^{2}$ is in the boundary of the solvency cone $\widehat{K}\left(\Pi\left(\omega_{n}\right)\right)$, for each $n \geq 0$, so that - up to a non-negative multiple - the only choice for $\left(\xi_{0}, \xi_{1}(\omega)\right)$, such that $\xi_{0}+\xi_{1}(\omega) \in \mathbb{R}_{+}^{4}$, for all $\omega \in \Omega$, is

$$
\xi_{0}=e_{1}-e_{2}, \quad \xi_{1}=e_{2}-e_{1}
$$

This shows that $\left(\Pi_{t}\right)_{t=0}^{1}$ satisfies the no arbitrage condition (NA), thus finishing the proof.

Remark 3.2 The above example does not satisfy the strict no arbitrage condition $\left(\mathrm{NA}^{\mathrm{s}}\right)$. Indeed, $\xi_{0}:=e^{1}-e^{2} \in \widehat{A}_{1}, \xi_{0} \in \widehat{K}\left(\Pi_{1}\left(\omega_{n}\right)\right)$, for $n \geq 0$, but, for $n \geq 2, \xi_{0}$ is not in the space $\widehat{F}\left(\Pi_{1}\left(\omega_{n}\right)\right)$ of "portfolios exchangeable to zero", which is in contradiction to condition $\left(\mathrm{NA}^{\mathrm{s}}\right)$ : it is indeed a crucial feature of the above construction that $e^{1}-e^{2} \in \widehat{K}\left(\Pi_{1}\left(\omega_{n}\right)\right)$, for all $n \geq 0$, but $-\left(e^{1}-e^{2}\right) \notin \widehat{K}\left(\Pi_{1}\left(\omega_{n}\right)\right)$, for $n \geq 2$.

However, there is a very shallow trick to adapt the above example to apply to the case of strict no arbitrage $\left(\mathrm{NA}^{\mathrm{s}}\right)$ too, by adding a fifth asset and one more time period.

Example 3.3 For $d=5$ and $T=2$, there is an $\mathbb{R}^{d}$-valued bid-ask process $\left(\widetilde{\Pi}_{t}\right)_{t=0}^{2}$ satisfying the strict no arbitrage condition $\left(N A^{s}\right)$, but such that

$$
\bar{A}_{T} \cap L^{0}\left(\Omega, \mathcal{F}, \mathbf{P} ; \mathbb{R}_{+}^{d}\right) \supsetneq\{0\} .
$$

Proof We choose the same stochastic base $\Omega=\left\{\omega_{0}, \omega_{1}, \ldots\right\}$ as above, let $\mathcal{F}_{2}=\mathcal{F}_{1}$ and adapt the previous Example 3.1, which we still denote by $\left(\Pi_{t}\right)_{t=0}^{1}$, to define the $5 \times 5$ matrices $\left(\widetilde{\Pi}_{t}\right)_{t=0}^{2}$.

For $t=0$, let $\widetilde{\pi}_{0}^{i j}=\pi_{0}^{i j}$, for $1 \leq i, j \leq 4$, and let $\widetilde{\pi}_{0}^{i 5}=\widetilde{\pi}_{0}^{5 i}=1+\varepsilon$, for all $i=1, \ldots, 4$.

For $t=1$ and $n=0$, define $\widetilde{\pi}_{1}^{i j}\left(\omega_{0}\right)=\pi_{1}^{i j}\left(\omega_{0}\right)$, for $1 \leq i, j \leq 4$, and $\widetilde{\pi}_{1}^{45}\left(\omega_{0}\right)=\widetilde{\pi}_{1}^{54}\left(\omega_{0}\right)=$ 1 , which defines $\widetilde{\Pi}_{1}\left(\omega_{0}\right)$ via (49). Similarly, for $t=1$ and $n=1$, we let $\widetilde{\pi}_{1}^{i j}\left(\omega_{1}\right)=1$, for all $1 \leq i, j \leq 5$.

For $t=1$ and $n \geq 2$, we have to take a little more care: let again $\widetilde{\pi}_{1}^{14}\left(\omega_{n}\right)=n$, $\widetilde{\pi}_{1}^{41}\left(\omega_{n}\right)=\frac{1}{n} ; \widetilde{\pi}_{1}^{23}\left(\omega_{n}\right)=n, \widetilde{\pi}_{1}^{32}\left(\omega_{n}\right)=\frac{1}{n}$; but now we let $\widetilde{\pi}_{1}^{43}\left(\omega_{n}\right)=\widetilde{\pi}_{1}^{34}\left(\omega_{n}\right)=1+\varepsilon$, while $\tilde{\pi}_{1}^{45}\left(\omega_{n}\right)=1, \widetilde{\pi}_{1}^{54}\left(\omega_{n}\right)=1+\varepsilon$, and $\widetilde{\pi}_{1}^{35}\left(\omega_{n}\right)=\widetilde{\pi}_{1}^{53}\left(\omega_{n}\right)=1+\varepsilon$. Applying again (49) this finishes the definition of $\widetilde{\Pi}_{1}\left(\omega_{n}\right)$.

For $t=2$ and $n=0,1$, we simply define $\widetilde{\Pi}_{2}\left(\omega_{n}\right)=\widetilde{\Pi}_{1}\left(\omega_{n}\right)$.

For $t=2$ and $n \geq 2$, we let $\widetilde{\pi}_{2}^{35}\left(\omega_{n}\right)=\widetilde{\pi}_{2}^{53}\left(\omega_{n}\right)=1$ and $\widetilde{\pi}_{2}^{i j}\left(\omega_{n}\right)=2 n$, for all other pairs $1 \leq i \neq j \leq 5$.

This finishes the definition of the bid-ask process $\left(\widetilde{\Pi}_{t}\right)_{t=0}^{2}$.

Next we show that $\bar{A}_{T} \cap L^{0}\left(\Omega, \mathcal{F}_{T}, \mathbf{P} ; \mathbb{R}_{+}^{d}\right) \supseteq\{0\}$ by constructing the increments of a "free lunch" $\left(\xi^{N}\right)_{N=1}^{\infty}=\left(\xi_{0}^{N}, \xi_{1}^{N}, \xi_{2}^{N}\right)_{N=1}^{\infty}$, similarly as in Example 3.1 above. For $t=0$, let again

$$
\xi_{0}^{N}=N\left(e^{1}-e^{2}\right)+\left(e^{3}-e^{4}\right) .
$$

For the case $n=0,1$, we also define $\xi_{1}^{N}\left(\omega_{n}\right)$ exactly as above, and let $\xi_{2}^{N}\left(\omega_{n}\right)=0$. 
For $n \geq 2$ and $t=1$, we define $\xi_{1}^{N}\left(\omega_{n}\right)$ in a slightly different way than in Example 3.1: for $2 \leq n \leq N$, let

$$
\begin{aligned}
\xi_{1}^{N}\left(\omega_{n}\right) & =-\frac{N}{n}\left(n e^{1}-e^{4}\right)-N\left(\frac{e^{3}}{n}-e^{2}\right)-\left(\frac{N}{n}-1\right)\left(e^{4}-e^{5}\right) \\
& =-N\left(e^{1}-e^{2}\right)-\left(e^{3}-e^{4}\right)-\left(\frac{N}{n}-1\right)\left(e^{3}-e^{5}\right),
\end{aligned}
$$

and, for $n>N$,

$$
\begin{aligned}
\xi_{1}^{N}\left(\omega_{n}\right) & =-\frac{N}{n}\left(n e^{1}-e^{4}\right)-N\left(\frac{e^{3}}{n}-e^{2}\right)-\left(\frac{N}{n}\right)\left(e^{4}-e^{5}\right) \\
& =-N\left(e^{1}-e^{2}\right)-\frac{N}{n}\left(e^{3}-e^{5}\right) .
\end{aligned}
$$

For $t=2$ and $2 \leq n \leq N$, define

$$
\xi_{2}^{N}\left(\omega_{n}\right)=\left(\frac{N}{n}-1\right)\left(e^{3}-e^{5}\right),
$$

and, for $n \geq N$,

$$
\xi_{2}^{N}\left(\omega_{n}\right)=\frac{N}{n}\left(e^{3}-e^{5}\right)
$$

Again one verifies that $\vartheta^{N}(\omega)=\xi_{0}^{N}+\xi_{1}^{N}(\omega)+\xi_{2}^{N}(\omega)$ converges pointwise to $\vartheta(\omega)$, such that $\vartheta\left(\omega_{0}\right)=\frac{e^{3}}{2}$ while $\vartheta\left(\omega_{n}\right)=0$, for $n \geq 1$, showing that $\bar{A}_{T} \cap L^{0}\left(\Omega, \mathcal{F}_{T}, \mathbf{P} ; \mathbb{R}_{+}^{d}\right) \supsetneq\{0\}$.

We still have to show that $\left(\widetilde{\Pi}_{t}\right)_{t=0}^{2}$ satisfies $\left(\mathrm{NA}^{\mathrm{s}}\right)$ : fix again the increments $\left(\xi_{0}, \xi_{1}(\omega), \xi_{2}(\omega)\right)$ of a self-financing portfolio process $\left(\vartheta_{0}, \vartheta_{1}(\omega), \vartheta_{2}(\omega)\right)$ sucht that $\vartheta_{2}(\omega) \geq$ 0 , for all $\omega \in \Omega$. The same argument as in Example 3.1 shows that $\xi_{0}$ is necessarily of the form $\xi_{0}=a\left(e^{1}-e^{2}\right)+b\left(e^{3}-e^{4}\right)$, with $a \geq 0, b \geq 0$.

To show that $b$ necessarily equals zero, fix $n \geq 2$ and consider the vector $w_{n}=$ $\left(\frac{1+\varepsilon}{n}, \frac{1}{n}, 1,1+\varepsilon, 1\right)$. Observe that $w_{n}$ is a consistent price system for $\Pi_{1}\left(\omega_{n}\right)$ as well as for $\Pi_{2}\left(\omega_{n}\right)$; this is easily checked by testing against the generators of the solvency cones $\widehat{K}\left(\Pi_{1}\left(\omega_{n}\right)\right)$ and $\widehat{K}\left(\Pi_{2}\left(\omega_{n}\right)\right)$. On the other hand, for $b>0$, we again have $\left(a\left(e^{1}-e^{2}\right)+\right.$ $\left.b\left(e^{3}-e^{4}\right), w_{n}\right)<0$, for sufficiently large $n$, which leads to a contradiction.

Hence we necessarily have $\vartheta_{0}=\xi_{0}=e^{1}-e^{2}$, up to a positive scalar, which we clearly may drop. But now - contrary to the previous Example 3.1 - for $n \geq 2$, the random variable $\vartheta_{0}\left(\omega_{n}\right) \equiv \vartheta_{0}$ does not take its values in the solvency cone $\widehat{K}\left(\widetilde{\Pi}_{1}\left(\omega_{n}\right)\right)$, i.e., we now have that $e^{1}-e^{2} \notin \widehat{K}\left(\widetilde{\Pi}_{1}\left(\omega_{n}\right)\right)$. Indeed, $u_{n}=\left(\frac{1}{n}, \frac{1+\varepsilon}{n}, 1+\varepsilon, 1,1\right)$ is a consistent pricing system for $\widetilde{\Pi}_{1}\left(\omega_{n}\right)$, i.e., $u_{n}$ is non-negative on $\widehat{K}\left(\widetilde{\Pi}_{1}\left(\omega_{n}\right)\right)$. But $\left(e^{1}-e^{2}, u_{n}\right)<0$ showing that $e^{1}-e^{2} \notin \widehat{K}\left(\widetilde{\Pi}_{1}\left(\omega_{n}\right)\right)$.

Hence, for $t=1$, the strict no arbitrage condition (15) is satisfied.

For the case $t=2$ fix $n \geq 2$, and consider the cone $\widehat{K}\left(\widetilde{\Pi}_{1}\left(\omega_{n}\right), \widetilde{\Pi}_{2}\left(\omega_{n}\right)\right)$ which is generated by $\widehat{K}\left(\widetilde{\Pi}_{1}\left(\omega_{n}\right)\right)$ and $\widehat{\widehat{K}}\left(\widetilde{\Pi}_{2}\left(\omega_{n}\right)\right)$. One readily verifies that $y_{n}=\left(\frac{1}{n}, \frac{1}{n}, 1,1,1\right)$ is a functional, which vanishes on $\xi_{0}=e^{1}-e^{2}$, and is non-negative on $\widehat{K}\left(\widetilde{\Pi}_{1}\left(\omega_{n}\right), \widetilde{\Pi}_{2}\left(\omega_{n}\right)\right)$. Therefore, the only way to write $\xi_{0}$ as a linear combination with non-negative coefficients on the generators of $\widehat{K}\left(\widetilde{\Pi}_{1}\left(\omega_{n}\right)\right)$ and $\widehat{K}\left(\widetilde{\Pi}_{2}\left(\omega_{n}\right)\right)$ is to use those generators contained in the 
hyperplane $\left\{y_{n}=0\right\}$ of $\mathbb{R}^{5}$, which are $e^{1}-\frac{e^{4}}{n}, \frac{e^{3}}{n}-e^{2}, e^{4}-e^{5}$ and $\pm\left(e^{3}-e^{5}\right)$. A moment's reflection reveals that the only representation of $\xi_{0}=e^{1}-e^{2}$ as a linear combination with non-negative coefficients on these generators equals

$$
e^{1}-e^{2}=\left(e^{1}-\frac{e^{4}}{n}\right)+\left(\frac{e^{3}}{n}-e^{2}\right)+\frac{1}{n}\left(e^{4}-e^{5}\right)+\frac{1}{n}\left(e^{5}-e^{3}\right)
$$

Hence, for $\left(\xi_{0}, \xi_{1}(\omega), \xi_{2}(\omega)\right)$ as above, starting at $\xi_{0}=e^{1}-e^{2}$, we must have, for $n \geq 2$,

$$
\xi_{1}\left(\omega_{n}\right)=-\left(e^{1}-\frac{e^{4}}{n}\right)-\left(\frac{e^{3}}{n}-e^{2}\right)-\frac{1}{n}\left(e^{4}-e^{5}\right) \text { and } \xi_{2}\left(\omega_{n}\right)=-\frac{1}{n}\left(e^{5}-e^{3}\right) .
$$

Hence, for all $\xi_{0}, \xi_{1}(\omega)$ such that $\vartheta_{1}(\omega)=\xi_{0}+\xi_{1}(\omega) \in K\left(\Pi_{2}(\omega)\right)$, for all $\omega \in \Omega$, we have

$$
\vartheta_{1}\left(\omega_{n}\right) \in \widehat{F}\left(\Pi_{2}\left(\omega_{n}\right)\right), \text { for all } n \geq 0 .
$$

Indeed, for $n \geq 2$, we have shown this in (63), and for $n=0,1$ (64) is obvious.

Summing up, we have shown that $\left(\widetilde{\Pi}_{t}\right)_{t=0}^{2}$ satisfies the strict no arbitrage condition $\left(\mathrm{NA}^{\mathrm{s}}\right)$

Remark 3.4 In the two examples above we have shown that $\bar{A}_{T}$ intersects $L^{0}\left(\Omega, \mathcal{F}_{T}, \mathbf{P} ; \mathbb{R}_{+}^{d}\right)$ in a non-trivial way, where $\bar{A}_{T}$ is the closure of $\widehat{A}_{T}$ in the topology of $L^{0}\left(\Omega, \mathcal{F}_{T}, \mathbf{P} ; \mathbb{R}^{d}\right)$.

It is not hard to modify the example in such a way that $\bar{A}_{T}^{\infty}$ intersects $L^{\infty}\left(\Omega, \mathcal{F}_{T}, \mathbf{P} ; \mathbb{R}_{+}^{d}\right)$ in a non-trivial way, where $\bar{A}_{T}^{\infty}$ now denotes the closure of $\widehat{A}_{T} \cap$ $L^{\infty}\left(\Omega, \mathcal{F}_{T}, \mathbf{P} ; \mathbb{R}^{d}\right)$ with respect to the topology of uniform convergence. For example, it suffices to replace, for $n \geq 2$, the $\varepsilon$ 's in the definition of $\Pi_{1}\left(\omega_{n}\right)$ (resp. of $\widetilde{\Pi}_{1}\left(\omega_{n}\right)$ and $\left.\widetilde{\Pi}_{2}\left(\omega_{n}\right)\right)$ by $\frac{\varepsilon}{n}$. We leave the details to the energetic reader.

\section{The Super-Replication Theorem}

In this section we show a general super-replication theorem (Theorem 4.1 below) for contingent claims $\vartheta \in L^{0}\left(\Omega, \mathcal{F}_{T}, \mathbf{P} ; \mathbb{R}^{d}\right)$ generalizing Theorem 4.2 of [KRS 01]. In fact, Theorem 4.1 below is a rather straightforward corollary of the "abstract" result on the closedness of $\widehat{A}_{T}$ (Theorem 2.1 above); this is similar to the situation in the frictionless theory (compare [J 92], [AS 94], [DS 94], [DS 98]).

The theorem is related to the well-known results on the value of the super-replication problem for European options (compare [SSC 95], [LS 97], [CPT 99], [T 99], [BT 00]).

Theorem 4.1 Suppose that the bid-ask process $\left(\Pi_{t}\right)_{t=0}^{T}$ satisfies the robust no arbitrage condition $\left(N A^{r}\right)$.

For $\vartheta \in L^{0}\left(\Omega, \mathcal{F}, \mathbf{P} ; \mathbb{R}^{d}\right)$, and $v \in \mathbb{R}^{d}$ the following conditions are equivalent:

(i) There is a self financing portfolio process $\left(\vartheta_{t}\right)_{t=0}^{T}$ such that

$$
\vartheta \leq v+\vartheta_{T}
$$


(ii) For every consistent (or, equivalently, strictly consistent) pricing process $\left(Z_{t}\right)_{t=0}^{T}$ such that the negative part $\left(\vartheta, Z_{T}\right)_{-}$of the random variable $\left(\vartheta, Z_{T}\right)$ is integrable, we have

$$
\mathbf{E}\left[\left(\vartheta, Z_{T}\right)\right] \leq\left(v, Z_{0}\right)
$$

(ii') For every (or, equivalently, for some) $F_{T}$-measurable $[1, \infty[$-valued function $\psi$ satisfying $\psi \geq\|\vartheta\| \vee 1$, and every consistent (or, equivalently, strictly consistent) pricing process $\left(Z_{t}\right)_{t=0}^{T}$ such that $Z_{T} \in L_{\psi}^{\infty}\left(\Omega, \mathcal{F}_{T}, \mathbf{P} ; \mathbb{R}^{d}\right)$, we have

$$
\mathbf{E}\left[\left(\vartheta, Z_{T}\right)\right] \leq\left(v, Z_{0}\right)
$$

Proof $(\mathrm{i}) \Rightarrow$ (ii): We conclude from Remark 2.4 that, for $\vartheta_{T} \in \widehat{A}_{T}$ and $\left(Z_{t}\right)_{t=0}^{T}$ a consistent price system, the condition $\mathbf{E}\left[\left(\vartheta_{T}, Z_{T}\right)_{-}\right]<\infty$ implies that $\mathbf{E}\left[\left(\vartheta_{T}, Z_{T}\right)\right] \leq 0$.

Hence (65) implies that

$$
\begin{aligned}
\mathbf{E}\left[\left(\vartheta, Z_{T}\right)\right] & \leq \mathbf{E}\left[\left(v+\vartheta_{T}, Z_{T}\right)\right] \\
& =\left(v, Z_{0}\right)+\mathbf{E}\left[\left(\vartheta_{T}, Z_{T}\right)\right] \\
& \leq\left(v, Z_{0}\right)
\end{aligned}
$$

which shows that (ii) holds true in its strong version (i.e., for every consistent pricing process).

(ii') $\Rightarrow$ (i): Suppose that (ii') holds true in its weakest version, i.e., there is some $\psi \geq\|\vartheta\| \vee 1$ such that, for every strictly consistent pricing process $\left(Z_{t}\right)_{t=0}^{T}$ with $Z_{T} \in$ $L_{\varphi}^{\infty}\left(\Omega, \mathcal{F}_{T}, \mathbf{P} ; \mathbb{R}^{d}\right)$ we have

$$
\mathbf{E}\left[\left(\vartheta, Z_{T}\right)\right] \leq\left(v, Z_{0}\right) .
$$

To verify $(65)$ we shall show that $\vartheta-v \in \widehat{A}_{T} \cap L_{\varphi}^{1}\left(\Omega, \mathcal{F}_{T}, \mathbf{P} ; \mathbb{R}^{d}\right)$, where $\varphi=\psi^{-1}$. By Theorem 2.1, this set is a closed convex cone in $L_{\varphi}^{1}\left(\Omega, \mathcal{F}_{T}, \mathbf{P} ; \mathbb{R}^{d}\right)$. Hence, if $\vartheta-v$ is not in this set, we can find a separating continuous linear functional, i.e., $g \in L_{\varphi}^{\infty}\left(\Omega, \mathcal{F}_{T}, \mathbf{P} ; \mathbb{R}^{d}\right)$ such that $\left.g\right|_{\widehat{A}_{T} \cap L_{\varphi}^{1}\left(\Omega, \mathcal{F}_{T}, \mathbf{P} ; \mathbb{R}^{d}\right)} \leq 0$, while $(\vartheta-v, g)>0$. By Lemma 2.5 we have that $Z_{T}:=\mathbf{E}\left[g \mid \mathcal{F}_{t}\right]$ defines a consistent price system $\left(Z_{T}\right)_{t=0}^{T}$, for which we then have

$$
\mathbf{E}\left[\left(\vartheta, Z_{T}\right)\right]>\left(v, Z_{0}\right) .
$$

This is not yet a contradiction to our assumption, as $\left(Z_{t}\right)_{t=0}^{T}$ is not necessarily strictly consistent. For this last step, let $\left(Z_{t}^{s}\right)_{t=0}^{T}$ be any strictly consistent price system such that $Z_{T}^{s} \in L_{\varphi}^{\infty}\left(\Omega, \mathcal{F}_{T}, \mathbf{P} ; \mathbb{R}^{d}\right)$; the existence of such a process is guaranteed by Theorem 1.7 and Remark 2.2.

For $0<\varepsilon \leq 1$, the process $\left(Z_{t}^{\varepsilon}\right)_{t=0}^{T}=\left(\varepsilon Z_{t}^{s}+Z_{t}\right)_{t=0}^{T}$ is a strictly consistent price process too. It is straightforward to check that, for $\varepsilon>0$ sufficiently small, we have

$$
\mathbf{E}\left[\left(\vartheta, Z_{T}^{\varepsilon}\right)\right]>\left(v, Z_{0}^{\varepsilon}\right),
$$

which yields the desired contradiction.

(ii) $\Rightarrow$ (ii'): This is the obvious implication, showing in particular that the two versions of (ii) and the four versions of (ii') all are equivalent. 
Remark 4.2 The message of the theorem is quite intuitive: roughly speaking, a portfolio $v \in \mathbb{R}^{d}$ is sufficient as initial endowment to superreplicate the contingent claim $\vartheta$, iff for each consistent price system $\left(Z_{t}\right)_{t=0}^{T}$, the price $\left(v, Z_{0}\right)$ at time $t=0$ is at least as big as the expected price $\mathbf{E}\left[\left(\vartheta, Z_{T}\right)\right]$ at time $t=T$.

Of course, to make this statement precise, we have - at least - to make sure that the latter expectation exists (which, in general, need not be the case). A pleasant feature of the above theorem is that the mild assumption (ii) guarantees already the validity of the above statement, without imposing any additional integrability or boundedness conditions on $\vartheta$.

For example, in [KRS 01, Theorem 4.2] $\vartheta$ is assumed to be uniformly bounded from below (in the sense that $\vartheta \geq-c \mathbf{1}$, for some $c>0$ ). This setting is a special case of the above Theorem 4.1, as for such a contingent claim $\vartheta$, the negative part $\left(\vartheta, Z_{T}\right)_{-}$is integrable, for each consistent pricing process $\left(Z_{t}\right)_{t=0}^{T}$. Hence we then have that $(65)$ holds true iff (66) holds true for all consistent pricing systems $\left(Z_{t}\right)_{t=0}^{T}$.

\section{A Appendix}

In this section we assemble some auxiliary results of a technical - mainly measuretheoretic - nature. In particular we use the nice idea of random subsequences, which was used by Y. Kabanov and C. Stricker [KS 01a] in the present context (these authors give credit to H.-J. Engelbert and H. v. Weizsäcker for Lemma 2 in [KS01a]). This notion replaces in a more elegant way the arguments of Komlos-type relying on convex combinations, as used, e.g., in [S 92].

Definition A.1 Fix a probability space $(\Omega, \mathcal{F}, \mathbf{P})$. A strictly increasing sequence $\left(\tau_{k}\right)_{k=1}^{\infty}$ of $\mathbf{N}$-valued, $\mathcal{F}$-measurable random variables is called an increasing random sequence.

Given two increasing random sequences $\left(\tau_{k}^{1}\right)_{k=1}^{\infty}$ and $\left(\tau_{k}^{2}\right)_{k=1}^{\infty}$, we say that $\left(\tau_{k}^{2}\right)_{k=1}^{\infty}$ is a random subsequence of $\left(\tau_{k}^{1}\right)_{k=1}^{\infty}$ iff, for almost each $\omega \in \Omega$, the sequence $\left(\tau_{k}^{2}(\omega)\right)_{k=1}^{\infty}$ is a subsequence of $\left(\tau_{k}^{1}(\omega)\right)_{k=1}^{\infty}$ or, equivalently, if there is an increasing random sequence $\left(\pi_{k}\right)_{k=1}^{\infty}$ such that $\tau_{k}^{2}=\tau_{\pi_{k}}^{1}$.

We remark that - just as in usual analysis - one may take a random subsequence of a random subsequence to obtain another random subsequence. A similar remark applies to the notion of the extraction of a diagonal subsequence of an increasing sequence of random subsequences.

We now reformulate Lemma 2 of [KS 01a] and, for the convenience of the reader, we also give a proof.

Lemma A.2 For a sequence $\left(f_{n}\right)_{n=1}^{\infty}=\left(f_{n}^{1}, \ldots, f_{n}^{d}\right) \in L^{0}\left(\Omega, \mathcal{F}, \mathbf{P} ; \mathbb{R}^{d}\right)$ there is a random subsequence $\left(\tau_{k}\right)_{k=1}^{\infty}$ such that the sequence of random variables $\left(g_{k}(\omega)\right)_{k=1}^{\infty}=\left(f_{\tau_{k}(\omega)}(\omega)\right)_{k=1}^{\infty}$ converges a.s. in the one-point-compactification $\mathbb{R}^{d} \cup\{\infty\}$ to a random variable $f \in$ $L^{0}\left(\Omega, \mathcal{F}, \mathbf{P} ; \mathbb{R}^{d} \cup\{\infty\}\right)$.

In fact, we may find the subsequence such that

$$
\|f(\omega)\|_{\mathbb{R}^{d}}=\limsup _{n \rightarrow \infty}\left\|f_{n}(\omega)\right\|_{\mathbb{R}^{d}}, \quad \text { a.s. }
$$

where $\|\infty\|_{\mathbb{R}^{d}}=\infty$. 
Proof Define inductively the increasing random sequence $\left(\tau_{k}^{0}\right)_{k=1}^{\infty}$ by $\tau_{1}^{0}=1$ and

$$
\tau_{k}^{0}(\omega)=\inf \left\{n: n>\tau_{k-1}^{0}(\omega) \text { and }\left\|f_{n}(\omega)\right\|_{\mathbb{R}^{d}}>\min \left(k, \limsup _{j \rightarrow \infty}\left\|f_{j}(\omega)\right\|_{\mathbb{R}^{d}}-\frac{1}{k}\right)\right\} \text {. }
$$

Clearly we then have

$$
\lim _{k \rightarrow \infty}\left\|f_{\tau_{k}^{0}(\omega)}(\omega)\right\|_{\mathbb{R}^{d}}=\limsup _{n \rightarrow \infty}\left\|f_{n}(\omega)\right\|_{\mathbb{R}^{d}}
$$

Next we define $\tau_{1}^{1}=1$ and

$$
\tau_{k}^{1}=\inf \left\{\tau_{n}^{0}(\omega): \tau_{n}^{0}(\omega)>\tau_{k-1}^{1}(\omega) \text { and } f_{\tau_{n}^{0}}^{1}(\omega)>\min \left(k, \limsup _{j \rightarrow \infty} f_{\tau_{j}^{0}(\omega)}^{1}(\omega)-\frac{1}{k}\right)\right\}
$$

The random subsequence $\left(f_{\tau_{k}^{1}}\right)_{k=1}^{\infty}$ then still satisfies equality (76) and, in addition, the first coordinate $\left(f_{\tau_{k}^{1}}\right)_{k=1}^{\infty}$ converges almost surely in the one-point compactification of $\mathbb{R}$. Repeating in an obvious way this extraction of random subsequences $d$ times, we arrive at a random subsequence $\left(\tau_{k}\right)_{k=1}^{\infty}=\left(\tau_{k}^{d}\right)_{k=1}^{\infty}$ satisfying the assertions of the lemma.

Remark A.3 If we start with a random sequence $\left(f_{\pi_{n}}\right)_{n=1}^{\infty}$ instead of the sequence $\left(f_{n}\right)_{n=1}^{\infty}$, then we clearly may again find a random subsequence $\left(f_{\tau_{n}}\right)_{n=1}^{\infty}$, of $\left(f_{\pi_{n}}\right)_{n=1}^{\infty}$, with the indicated properties.

We have formulated and proved the above lemma in the form which corresponds precisely to what we need for the present applications. But it is clear, that the lemma may also be formulated in greater generality: for example, we may replace the compact space $\mathbb{R}^{d} \cup\{\infty\}$ by an arbitrary compact metric space $\widehat{K}$, or even by a family $\left(\widehat{K}_{\omega}\right)_{\omega \in \Omega}$ of compact, metric spaces, depending in a measurable way on $\omega \in \Omega$.

Lemma A.4 Let $\mathcal{N}$ be a linear subspace of $L^{0}\left(\Omega, \mathcal{F}, \mathbf{P} ; \mathbb{R}^{d}\right)$, with the following properties

(i) $\mathcal{N}$ is closed under multiplication with scalar-valued $\mathcal{F}$-measurable functions, i.e., for $f \in \mathcal{N}$ and $h \in L^{0}(\Omega, \mathcal{F}, \mathbf{P} ; \mathbb{R})$, we have $h f \in \mathcal{N}$.

(ii) $\mathcal{N}$ has the following monotonicity property: for $f \in L^{0}\left(\Omega, \mathcal{F}, \mathbf{P} ; \mathbb{R}^{d}\right)$ and an increasing sequence $\left(D_{n}\right)_{n=1}^{\infty} \in \mathcal{F}$, such that $\lim _{n \rightarrow \infty} \mathbf{P}\left[D_{n}\right]=1$, and $f \chi_{D_{n}} \in \mathcal{N}$, for all $n \geq 1$, we have that $f \in \mathcal{N}$.

Then there is an a.s. unique $\mathcal{F}$-measurable map $\omega \mapsto N_{t}(\omega)$, taking its values in the linear subspaces of $\mathbb{R}^{d}$, such that, for $f \in L^{0}\left(\Omega, \mathcal{F}, \mathbf{P} ; \mathbb{R}^{d}\right)$, we have that $f \in \mathcal{N}$ iff $f(\omega) \in N_{t}(\omega)$ almost surely. In particular, $\mathcal{N}$ is closed.

Proof For every countable subset $\left(f_{i}\right)_{i \in I}$ of $\mathcal{N}$, define the function

$$
\operatorname{rk}\left(\left(f_{i}\right)_{i \in I}\right)(\omega)=\operatorname{dim}\left(\operatorname{span}_{i \in I} f_{i}(\omega)\right) .
$$

The function $\operatorname{rk}\left(\left(f_{i}\right)_{i \in I}\right)$ is well-defined, $\mathcal{F}$-measurable, and $\{0, \ldots, d\}$-valued. We denote by $\operatorname{rk}(\omega)$ the essential supremum of the family of functions $\operatorname{rk}\left(\left(f_{i}\right)_{i \in I}\right)(\omega)$, where 
$\left(f_{i}\right)_{i \in I}$ runs through all countable subsets of $\mathcal{N}$. It is straightforward to verify that there is a sequence $\left(f_{n}\right)_{n=1}^{\infty} \in \mathcal{N}$ such that

$$
\operatorname{rk}(\omega)=\operatorname{rk}\left(\left(f_{n}\right)_{n=1}^{\infty}\right)(\omega), \quad \text { a.s. }
$$

Next we define, for $\omega \in \Omega$, the sequence $\left(n_{i}(\omega)\right)_{i=1}^{\mathrm{rk}(\omega)}$, in the following way: $n_{1}(\omega)$ is the first $n$ such that $f_{n}(\omega) \neq 0 ; n_{2}(\omega)$ is the first $n>n_{1}(\omega)$ such that $f_{n}(\omega)$ is linearly independent of $f_{n_{1}(\omega)}$; continuing in an obvious way, $n_{\operatorname{rk}(\omega)}$ is the first $n>n_{\operatorname{rk}(\omega)-1}$ such that $f_{n}(\omega)$ is linearly independent of $f_{n_{1}}(\omega) \ldots, f_{n_{\mathrm{rk}(\omega)-1}}(\omega)$. Clearly the functions $n_{1}(\omega), \ldots, n_{d}(\omega)$ are $\mathcal{F}$-measurable, if we define $n_{i}(\omega)=\infty$, for $i>\operatorname{rk}(\omega)$.

We now define $N_{t}(\omega)=\operatorname{span}\left(f_{n}(\omega)\right)_{n=1}^{\infty}=\operatorname{span}\left(f_{n_{i}(\omega)}(\omega)\right)_{i=1}^{\mathrm{rk}(\omega)}$, to obtain an $\mathcal{F}$ measurable function taking values in the set of linear subspaces of $\mathbb{R}^{d}$ (equipped with its natural Borel-structure, when considering subspaces of $\mathbb{R}^{d}$ as elements of the corresponding Grassmannian manifold). Clearly, for $f \in \mathcal{N}$, we have that $f(\omega) \in N_{t}(\omega)$, almost surely.

To show that the converse also holds true, define $\left(g_{i}\right)_{i=1}^{d}$ by

$$
g_{i}=\sum_{n=1}^{\infty} f_{n} \chi_{\left\{n_{i}(\omega)=n\right\}} .
$$

From assumptions (i) and (ii) we conclude that the functions $g_{i}$ are elements of $\mathcal{N}$, for $i=1, \ldots, d$, and clearly $\left(g_{i}(\omega)\right)_{i=1}^{\mathrm{rk}(\omega)}$ a.s. spans $N_{t}(\omega)$, while $g_{i}(\omega)=0$, for $\operatorname{rk}(\omega)<i \leq d$.

Let $f \in L^{0}\left(\Omega, \mathcal{F}, \mathbf{P} ; \mathbb{R}^{d}\right)$ be such that $f(\omega) \in N_{t}(\omega)$ almost surely. We want to show that $f \in \mathcal{N}$. For almost each $\omega$, there are unique coefficients $\left(a_{i}(\omega)\right)_{i=1}^{d}$ such that $a_{i}(\omega)=0$ for $\operatorname{rk}(\omega)<i \leq d$, and

$$
f(\omega)=\prod_{i=1}^{d} a_{i}(\omega) g_{i}(\omega), \text { a.s. }
$$

Clearly the functions $a_{i}(\omega)$ are $\mathcal{F}$-measurable. Hence we conclude from assumption (i) that $f$ is in $\mathcal{N}$.

Finally we show an elementary result, relating the notions of "strictly consistent price systems" and "smaller bid-ask spreads".

Proposition A.5 Let $\Pi$ be a $d \times d$ bid-ask matrix. A vector $w \in \mathbb{R}^{d}$ is a strictly consistent price system for $\Pi$, iff there is a bid-ask matrix $\widetilde{\Pi}$ with smaller bid-ask spreads than $\Pi$, such that $w$ is a consistent price system for $\widetilde{\Pi}$.

Proof It is convenient to reduce the situation to the case, where $\Pi$ satisfies the "efficient friction condition" (see [KRS 01] for the definition):

We call $i$ equivalent to $j$ if $\pi^{i j} \pi^{j i}=1$. It is straight-forward to check that this is indeed an equivalence relation on $\{1, \ldots, d\}$. Choose respresentants $\left\{i_{1}, \ldots, i_{k}\right\}$ of each of the $k$ equivalence classes, write $\check{\Pi}$ for the $k \times k$ matrix $\left(\pi^{i_{p}, i_{q}}\right)_{1 \leq p, q \leq k}$, and denote by $\widehat{K}^{*}(\check{\Pi})$ the corresponding cone of consistent price systems in $\mathbb{R}^{k}$.

It is straight-forward to check that the canonical projection $p: \mathbb{R}^{d} \rightarrow$ $\mathbb{R}^{k}, p\left(w^{1}, \ldots, w^{d}\right)=\left(w^{i_{1}}, \ldots, w^{i_{k}}\right)$ induces an isomorphism between $\widehat{K}^{*}(\Pi)$, and $\widehat{K}^{*}(\check{\Pi})$ with respect to their topological as well as their affine structure.

Hence $w \in \mathbb{R}^{d}$ is in the relative interior of $\widehat{K}^{*}(\Pi)$, iff $p(w)$ is in the relative interior of $\widehat{K}^{*}(\check{\Pi})$. The above construction is done in such a way, that $\widehat{K}^{*}(\check{\Pi})$ has nonempty interior 
in $\mathbb{R}^{k}$, so that the latter condition is equivalent to $p(w)$ being in the interior of $\widehat{K}^{*}(\check{\Pi})$. Similarly, one verifies that $\Pi$ admits a bid-ask matrix $\widetilde{\Pi}$ with smaller bid-ask spreads than $\Pi$, such that $w$ is a consistent price system for $\widetilde{\Pi}$ iff the same assertion is true for $\check{\Pi}$ and $p(w)$.

Summing up this reduction step, we may and do assume w.l.g. that $\pi^{i j} \pi^{j i}>1$, for all $1 \leq i \neq j \leq d$, in which case the relative interior of $\widehat{K}^{*}(\Pi)$ equals its interior.

$\widehat{K}^{*}(\Pi)$ is the intersection of $\mathbb{R}_{+}^{d}$ with the half-spaces $H^{i j} \subseteq \mathbb{R}^{d}$

$$
H^{i j}=\left\{w \in \mathbb{R}^{d}: \pi^{i j} w^{i}-w^{j} \geq 0\right\} .
$$

If $w$ is in the interior of $\widehat{K}^{*}(\Pi)$, than $\pi^{i j}>\frac{w^{j}}{w^{i}}$, for each $1 \leq i \neq j \leq d$. Defining the (frictionless) bid-ask matrix $\widetilde{\Pi}$ by

$$
\widetilde{\pi}^{i j}=\frac{w^{j}}{w^{i}}
$$

we obtain a bid-ask matrix $\widetilde{\Pi}$ with smaller bid-ask spreads then $\Pi$, such that $w$ is a consistent (in fact, a strictly consistent) price system for $\widetilde{\Pi}$.

Conversely suppose that there is $\widetilde{\Pi}$ with smaller bid-ask spreads then $\Pi$, such that $w$ is a consistent price system for $\widetilde{\Pi}$. By the above reduction step we have $\pi^{i j}>\frac{1}{\pi^{j i}}$, for all $1 \leq i \neq j \leq d$, so that $\pi^{i j}>\widetilde{\pi}^{i j}$. As $\widetilde{\pi}^{i j} w^{i}-w^{j} \geq 0$, for all $1 \leq i \neq j \leq d$, we can find a neighbourhood of $w$ which is contained in the intersection of $\mathbb{R}_{+}^{d}$ and the half-spaces $H^{i j}$ defined in (82), so that $w$ is in the interior of $\widehat{K}^{*}(\Pi)$.

\section{References}

[AS 94] J.P. Ansel, C. Stricker, (1994), Couverture des actifs contingents et prix maximum. Ann. Inst. Henri Poincaré, Vol. 30, pp. 303-315.

[BKT 01] B. Bouchard, Yu.M. Kabanov, N. Touzi, (2001), Option pricing by large risk aversion utility under transaction costs. Decisions in Economics and Finance, Vol. 24, No. 1, pp. 127-136.

[BT00] B. Bouchard, N. Touzi, (2000), Explicit solution of the multivariate superreplication problem under transaction costs. Annals of Applied Probability, Vol. 10, pp. 685-708.

[CK 96] J. Cvitanic, I. Karatzas, (1996), Hedging and portfolio optimization under transaction costs: A martingale approach. Mathematical Finance, Vol. 6, No. 2, pp. $133-165$.

[CPT 99] J. Cvitanic, H. Pham, N. Touzi, (1999), A closed-form solution to the problem of super-replication under transaction costs. Finance and Stochastics, Vol. 3, pp. 3554.

[DMW 90] R.C. Dalang, A. Morton, W. Willinger, (1990), Equivalent martingale measures and no-arbitrage in stochastic. Stochastics and Stochastics Reports, Vol. 29, pp. 185-201. 
[DN 90] M.H.A. Davis, A. Norman, (1990), Portfolio selection with transaction costs. Math. Operation Research, Vol. 15, pp. 676-713.

[De 92] F. Delbaen, (1992), Representing martingale measures when asset prices are continuous and bounded. Mathematical Finance, Vol. 2, pp. 107-130.

[DKV 02] F. Delbaen, Yu.M. Kabanov, E. Valkeila (2002), Hedging under transaction costs in currency markets: a discrete-time model. Mathematical Finance, Vol. 12, No. 1.

[DS 94] F. Delbaen, W. Schachermayer, (1994), A General Version of the Fundamental Theorem of Asset Pricing. Math. Annalen, Vol. 300, pp. 463-520.

[DS 98] F. Delbaen, W. Schachermayer, (1998), The Fundamental Theorem of Asset Pricing for Unbounded Stochastic Processes. Mathematische Annalen, Vol. 312, pp. 215-250.

[DH 86] D. Duffie, C.F. Huang, (1986), Multiperiod security markets with differential information; martingales and resolution times. J. Math. Econom., Vol. 15, pp. 283303.

[ET 00] I. Evstigneev, M. Taksar, (2000), A general framework for arbitrage pricing and hedging theorems in models of financial markets. Preprint.

[FH 97] B. Flesaker, L.P. Hughston, (1997), International Models for Interest Rates and Foreign Exchange. Net Exposure, Vol. 3, pp. 55-79.

[HK 79] J.M. Harrison, D.M. Kreps, (1979), Martingales and arbitrage in multiperiod securities markets. J. Econ. Theory, Vol. 20, pp. 381-408.

[HP 81] J.M. Harrison, S.R. Pliska, (1981), Martingales and stochastic integrals in the theory of continuous trading. Stochastic Processes \& Applications, Vol. 11, pp. 215260.

[J 92] S.D. Jacka, (1992), A martingale representation result and an application to incomplete financial markets. Mathematical Finance, Vol. 2, pp. 239-250.

[JK 95a] E. Jouini, H. Kallal, (1995), Martingales and arbitrage in securities markets with transaction costs. Journal of Economic Theory, Vol. 66, pp. 178-197.

[JK 95b] E. Jouini, H. Kallal, (1995), Arbitrage in securities markets with short-sales constraints. Mathematical Finance, Vol. 3, pp. 237-248.

[JK 99] E. Jouini, H. Kallal, (1999), Viability and Equilibrium in Securities Markets with Frictions. Mathematical Finance, Vol. 9, No. 3, pp. 275-292.

[K 99] Yu.M. Kabanov, (1999), Hedging and liquidation under transaction costs in currency markets. Finance and Stochastics, Vol. 3, No. 2, pp. 237-248.

[K 01] Yu.M. Kabanov, (2001), Arbitrage Theory. Handbooks in Mathematical Finance. Option Pricing: Theory and Practice, pp. 3-42. 
[KK 94] Yu.M. Kabanov, D. Kramkov, (1994), No arbitrage and equivalent martingale measures: An elementary proof of the Harrison-Pliska theorem. Theory Prob. Appl., Vol. 39.

[KL 02] Yu.M. Kabanov, G. Last, (2002), Hedging under transaction costs in currency markets: a continuous-time model. Mathematical Finance, Vol. 12, No. 1.

[KS 01a] Yu.M. Kabanov, Ch. Stricker, (2001), A teachers' note on no-arbitrage criteria. Lecture Notes in Mathematics, Vol. 1755, pp. 149-152.

[KS 01b] Yu.M. Kabanov, Ch. Stricker, (2001), The Harrison-Pliska arbitrage pricing theorem under transaction costs. Journal of Mathematical Economics, Vol. 35, No. 2, pp. 185-196.

[KRS01] Yu.M. Kabanov, M. Rásonyi, Ch. Stricker, (2001), No-arbitrage criteria für financial markets with efficient friction. Preprint, to appear in Finance and Stochastics.

[KRS 02] Yu.M. Kabanov, M. Rásonyi, Ch. Stricker, (2002), On the closedness of sums of convex cones in $L^{0}$ and the robust no-arbitrage property. Preprint.

[KPT 99] P.-F. Koehl, H. Pham, N. Touzi, (1999), On super-replication under Transaction costs in general discrete-time models. Theory of Probability and its Applications, Vol. 45, pp. 783-788.

[K 81] D.M. Kreps, (1981), Arbitrage and equilibrium in economies with infinitely many commodities. Journal of Mathematical Economics, Vol. 8, pp. 15-35.

[LW 01] S.F. LeRoy, J. Werner, (2001), Principles of Financial Economics. Cambridge University Press.

[LS 97] S. Levental, A.V. Skorohod, (1997), On the possibility of hedging options in the presence of transaction costs. Annals of Applied Probability, Vol. 7, pp. 410-443.

[PT 99] H. Pham, N. Touzi, (1999), The fundamental theorem of asset pricing with cone constraints. Journal of Mathematical Economics, Vol. 31, pp. 265-279.

[P 01] I. Penner, (2001), Arbitragefreiheit in Finanzmärkten mit Transaktionskosten. Diploma Thesis at the Humboldt University Berlin.

[R 94] L.C.G. Rogers, (1994), Equivalent martingale measures and no-arbitrage. Stochastics and Stochastic Reports, Vol. 51, pp. 41-49.

[S 92] W. Schachermayer, (1992), A Hilbert space proof of the fundamental theorem of asset pricing in finite discrete time. Insurance: Mathematics and Economics, Vol. 11, pp. 249-257.

[S 94] W. Schachermayer, (1994), Martingale Measures for discrete time processes with infinite horizon. Math. Finance, Vol. 4, No. 1, pp. 25-55. 
[S 01] W. Schachermayer, (2001), Introduction to the Mathematics of Financial Markets. Preprint, to appear in Springer Lecture Notes (St. Flour summer school 2000).

[St 97] H. Strasser, (1997), On a Lemma of Schachermayer. Preprint, Vienna University of Economics and Business Administration (9 pages).

[St 90] C. Stricker, (1990), Arbitrage et lois de martingale. Ann. Inst. H.Poincaré Probab. Statist., Vol. 26, pp. 451-460.

[SSC 95] H.M. Soner, S.E. Shreve, J. Cvitanic, (1995), There is no nontrivial hedging portfolio for option pricing with transaction costs. Annals of Applied Probability, Vol. 5, pp. 327-355.

[T 99] N. Touzi, (1999), Super-replication under proportional transaction costs: from discrete to continuous-time models. Mathematical Methods of Operation Research, Vol. 50, pp. 297-320.

[Y 80] J.A. Yan, (1980), Characterisation d' une classe d'ensembles convexes de $L^{1}$ ou $H^{1}$. Lect. Notes Mathematics, Vol. 784, pp. 220-222.

\section{Walter Schachermayer}

Department of Financial and Actuarial Mathematics

Vienna University of Technology

Wiedner Hauptstrasse 8-10 / 105

A-1040 Vienna

Austria

wschach@fam.tuwien.ac.at

http://www.fam.tuwien.ac.at/〜 wschach/ 